\title{
ELDOR-detected NMR at Q-band
}

\section{Thilo Hetzke ${ }^{1}$, Alice M. Bowen ${ }^{1,2}$, Thomas F. Prisner ${ }^{1}$}

${ }^{1}$ Institute of Physical and Theoretical Chemistry and Center of Biomolecular Magnetic Resonance, Goethe University Frankfurt, Frankfurt am Main, Germany, ${ }^{2}$ Center for Advanced Electron Spin Resonance (CAESR), Department of Chemistry, University of Oxford, Oxford, United Kingdom

In recent years, Electron-electron double resonance detected NMR (EDNMR) has gained considerable attention as a pulsed EPR technique to probe hyperfine interactions. Most experiments published so far were performed at W-band frequencies or higher, as at lower frequencies detection of weakly coupled low- $\gamma$ nuclei is hampered by the presence of a central blind spot, which occurs at zero frequency. In this article we show that EDNMR measurements and a meaningful data analysis is indeed possible at intermediate microwave frequencies (Q-band, $34 \mathrm{GHz}$ ), once experimental parameters have been optimized. With highly selective detection pulses and Gaussian shaped ELDOR pulses it is possible to detect low- $\gamma$ nuclei coupled to paramagnetic $\mathrm{Mn}^{2+}$. Weakly coupled ${ }^{14} \mathrm{~N}$ resonances, which are separated from the zero frequency by only $2.8 \mathrm{MHz}$, were readily detected. In systems where different spin active nuclei are coupled to the electron spin, particular care has to be taken when using higher-powered ELDOR pulses, as combination frequencies from the two nuclei $\left(\Delta m_{\mathrm{S}}= \pm 1, \Delta m_{\mathrm{l}, 1}= \pm 1, \Delta m_{\mathrm{l}, 2}= \pm 1\right)$ can lead to severe line broadening and complicated EDNMR spectra. We also compare the EDNMR spectra of ${ }^{13} \mathrm{C}$-labeled Mn-DOTA to ${ }^{13} \mathrm{C}$-Mims ENDOR to get a better insight into the similarities and differences in the results of the two techniques for ${ }^{13} \mathrm{C}$ hyperfine coupling.

\section{Introduction}

Electron-electron double resonance detected NMR (ELDOR-detected NMR or just EDNMR) is a technique of pulsed EPR spectroscopy to measure hyperfine couplings of paramagnetic spin centers to nearby spin-active nuclei. First proposed by the group of Arthur Schweiger in 1994 [1], it took approximately 10 more years until it found further scientific applications and became a useful tool for research [2-4]. 
As the technique has gained wider applications several EDNMR review articles have been published, describing the experiment in detail [5-8]. In this article, we will therefore only give a short introduction to EDNMR. A more detailed theoretical description can be found in the aforementioned references. A typical EDNMR pulse sequence consists of two microwave channels, a detection channel ( $\left.v_{\text {det }}\right)$ and an ELDOR channel ( $V E L D)$. The detection channel consists of a Hahn-echo sequence and is operated at a fixed frequency to monitor the intensity of an allowed EPR transition. The second microwave channel consists of a single, typically long microwave pulse referred to as ELDOR or high-turning angle (HTA) pulse. VELD of the ELDOR pulse is normally swept symmetrically around $v$ det. Every time the ELDOR pulse hits a formally forbidden transition of the type $\Delta m_{\mathrm{s}}= \pm 1, \Delta m_{\mathrm{l}}= \pm 1$ (or for high spin nuclei also $\Delta m_{1}= \pm 2, \pm 3$, etc.) population from the lower state is transferred to an excited state. The removal of population from the lower state of the allowed transition manifests itself as a reduction of intensity of the integrated spin echo in $v_{\text {det}}$, as the population difference of the allowed transition is reduced. As $v_{E L D}$ approaches $v_{\text {det, }}$, the ELDOR pulse saturates the detected EPR transition. As a consequence, the integrated intensity drops to zero at $\Delta v=v_{E L D}-v_{\text {det }}=0$. This is commonly referred to as the central blind spot. The unit of $\Delta v$ is typically given in $\mathrm{MHz}$. Weakly coupled ligand nuclei resonate at their respective Larmor frequency $u$, with individual peaks split by the hyperfine coupling constant $A_{\text {I. }}$. These nuclei yield a symmetric EDNMR spectrum with respect to the central blind spot, as allowed EPR transitions in all nuclear spin manifolds are excited simultaneously. Strongly coupled nuclei resonate at $A_{1} / 2$ with peaks being split by $2 u$ to first order. The EDNMR spectrum may be asymmetric with respect to the central blind spot, if the allowed EPR transitions in the different nuclear manifolds can be selectively excited, as demonstrated here for various $\mathrm{Mn}^{2+}$ complexes.

The central blind spot is the reason why most of the currently published EDNMR studies have been performed at high microwave frequencies (W-band, $95 \mathrm{GHz}$ and $3393 \mathrm{mT}$ for $g=2$ ). At these field strengths weakly coupled nuclei, especially those of low- $\gamma$ nuclei, have sufficiently high Larmor frequencies such that they are no longer obscured by the central blind spot of the experiment. Examples include work by Jeschke and Spiess [2], work by Sun Un and coworkers on $\mathrm{Mn}^{2+}$ systems [9-11], work 
by the Goldfarb lab [12-15], the Lubitz lab $[4,16,17]$ and the van Doorslaer lab [1820].

To the authors knowledge, only few EDNMR studies at lower microwave frequencies have so far been reported. Examples include ${ }^{1} \mathrm{H}$-EDNMR at X-band [3], strongly coupled ${ }^{61} \mathrm{Ni}-E D N M R$ at Q-band [21], strongly coupled ${ }^{14} \mathrm{~N}-E D N M R$ at Q-band [22] and a Q-band EDNMR study on copper single crystals [23]. All these studies, however, exclude the investigation of weakly coupled low- $\gamma$ nuclei such as ${ }^{14} \mathrm{~N}$ at these lower microwave frequencies and field strengths. In this work, it is shown that useful information can be extracted from Q-band EDNMR data of $\mathrm{Mn}^{2+}$ model systems, even for low- $\gamma$ nuclei such as ${ }^{14} \mathrm{~N}$ and ${ }^{17} \mathrm{O}$, once the experimental parameters have been carefully optimized.

\section{Q-band EDNMR versus high-field EDNMR}

High-field EDNMR comes with two major advantages the first one being that weakly coupled low- $\gamma$ nuclei are well separated from the central blind spot. For example; the biologically relevant ${ }^{14} \mathrm{~N}$ nucleus resonates at $10.2 \mathrm{MHz}$ at $\mathrm{W}$-band frequencies, this means that weakly coupled ${ }^{14} \mathrm{~N}$ nuclei will only overlap slightly with the Lorentzian shaped central blind spot function, which has a FWHM (full width half maximum) of typically $6 \mathrm{MHz}$ [5]. Secondly, different nuclei are better resolved from one another. At W-band, the frequency difference between ${ }^{23} \mathrm{Na}(v 23 \mathrm{Na}=37.8 \mathrm{MHz})$ and ${ }^{13} \mathrm{C}$ $\left(v_{13 \mathrm{C}}=35.9 \mathrm{MHz}\right)$ is $1.9 \mathrm{MHz}$, whereas at Q-band, the frequency difference is reduced to $0.7 \mathrm{MHz}\left(v_{23} \mathrm{Na}=13.5 \mathrm{MHz}, v_{13} \mathrm{C}=12.8 \mathrm{MHz}\right)$. Although the resolution of EDNMR at $\mathrm{Q}$-band is accurate enough to tell if predominantly ${ }^{23} \mathrm{Na}$ or ${ }^{13} \mathrm{C}$ is present in the sample, it is difficult to give a 'quantitative' answer, as it is impossible to separate ${ }^{23} \mathrm{Na}$ and ${ }^{13} \mathrm{C}$ signals. Although ${ }^{23} \mathrm{Na}$ and ${ }^{13} \mathrm{C}$ signals are still not fully separated at $\mathrm{W}$-band, the larger separation facilitates a more quantitative treatment [10]. All Larmor frequencies given in this article are based on $g=2$ and $v_{0}=33.5 \mathrm{GHz}, B_{0}=1200 \mathrm{mT}$ and $v_{0}=94.0 \mathrm{GHz}$, $B_{0}=3360 \mathrm{mT}$ for $\mathrm{Q}$ - and W-band respectively, unless calculated with reference to a specific example data set shown in a figure.

In contrast, an advantage of Q-band EDNMR over high-field EDNMR is, that forbidden transitions have an intrinsically higher transition probability $\left(I_{\mathrm{f}}\right)$ at $\mathrm{Q}$-band as $I_{\mathrm{f}}$ is inversely proportional to the square of the Larmor frequency $и$ [1]: 


$$
I_{a}=\frac{\left|v_{I}^{2}-\frac{1}{4}\left(v_{\alpha}-v_{\beta}\right)^{2}\right|}{v_{\alpha} v_{\beta}}
$$

and

$$
I_{f}=\frac{\left|v_{I}^{2}-\frac{1}{4}\left(v_{\alpha}+v_{\beta}\right)^{2}\right|}{v_{\alpha} v_{\beta}}
$$

Where $l_{\mathrm{a}}$ and $I_{\mathrm{f}}$ describe the transition probabilities of allowed and forbidden transitions, $\eta$ describes the nuclear Larmor frequency and $v_{\alpha}$ and $\nu_{\beta}$ the nuclear transitions in the different electron spin manifolds. If dictates the EDNMR intensity $h$ alongside with the nominal flip angle of the ELDOR pulse:

$$
h=1-I_{a} \cos \left(\beta_{0} \sqrt{I_{f}}\right)-I_{f} \cos \left(\beta_{0} \sqrt{I_{a}}\right)
$$

With $\beta \mathrm{f}=\omega \mathrm{ELD} t_{\mathrm{ELD}} \sqrt{l_{\mathrm{f}}}=\beta_{0} \sqrt{l_{\mathrm{f}} .} v_{\alpha}$ and $\nu_{\beta}$ are defined as:

$$
\left|v_{\alpha}\right|=\left[\left(v_{I}+A\right)^{2}+\left(\frac{1}{2} B\right)^{2}\right]^{1 / 2}
$$

and

$$
\left|v_{\beta}\right|=\left[\left(v_{I}-A\right)^{2}+\left(\frac{1}{2} B\right)^{2}\right]^{1 / 2}
$$

$A$ describes the secular part of the hyperfine coupling and contains the isotropic ( $\left.a_{\text {iso }}\right)$ as well as the dipolar hyperfine interaction $(T)$. $B$ describes the pseudosecular part of the hyperfine coupling and contains only the dipolar contribution of the hyperfine interaction [24]. Equations 1.1, 1.2, 1.4 and 1.5 are only valid for a $S=1 / 2, I=1 / 2$ spin system. For high-spin systems, the zero-field and quadrupole interactions lead to an additional level mixing of the spin states and therefore to an increase in $/ \mathrm{f}$. These highspin terms are also inversely proportional to $v^{2}$. As a consequence, their impact on $I_{\mathrm{f}}$ is larger at smaller magnetic fields [25,26]. For spin-1/2 nuclei such as ${ }^{15} \mathrm{~N}$ and ${ }^{31} \mathrm{P}$, which are known to have relatively small pseudosecular contributions to the hyperfine interaction Hamiltonian in $\mathrm{Mn}^{2+}$ coupled systems [27,28], If can become rather small, especially at higher fields. 
Another important aspect of EDNMR is its reproducibility, to allow comparison of different samples (e.g. different incubation times for $A B C$ transporters with $\mathrm{Mn}^{2+} / \mathrm{ATP}$ to investigate the transport cycle [29]). Reproducibility for EDNMR data is in large parts dictated by the effective $B_{1}$ field of the ELDOR pulse. This varies with the pulse frequency as it depends on the bandwidth profile of the resonator used. In our experiments, we have found that the Bruker EN 5170 D2 probehead is a robust resonator, which is easy to tune and gives similar bandwidth profiles for samples in similar solvent systems. An automatized nutation experiment with good resolution to probe the resonator bandwidth is routinely performed in under 10 minutes.

A further advantage of Q-band EDNMR is more of a practical nature: Q-band spectrometers are more widespread than W-band spectrometers. Additionally modern commercial Q-band spectrometers can be purchased with built-in AWG systems, this offers the possibility to easily use Gaussian shaped ELDOR pulses, which somewhat reduces the problem of the central blind spot (see Section 4.1).

\section{Methods}

\subsection{Sample Preparation}

For the $\mathrm{Mn}^{2+}$ hexaaqua complexes, an appropriate amount of $\mathrm{MnCl}_{2} \cdot 4 \mathrm{H}_{2} \mathrm{O}$ (SigmaAldrich) was dissolved in $\mathrm{ddH}_{2} \mathrm{O}$ to yield a $40 \mathrm{mM}$ stock solution. An aliquot of the stock solution was then further diluted with $\mathrm{ddH}_{2} \mathrm{O}$ or $\mathrm{H}_{2}{ }^{17} \mathrm{O}$ (Nukem Isotops, $>70 \% \mathrm{w} / \mathrm{w}$ isotope purity) to yield $4 \mathrm{mM} \mathrm{Mn}^{2+}$ solutions. Small amounts were then mixed with methanol $(50 \%, v / v)$ as glassing agent to yield a final $2 \mathrm{mM} \mathrm{Mn}^{2+}$ solution.

For Mn-DOTA complexes, an appropriate amount of DOTA (Strem Chemicals) or ${ }^{13} \mathrm{C}$-labeled DOTA was dissolved in a $100 \mathrm{mM}$ buffered triethanolamine solution at $\mathrm{pH}=7.5$ to yield a $4 \mathrm{mM}$ DOTA solution. $\mathrm{Mn}\left(\mathrm{ClO}_{4}\right)_{2} \cdot 4 \mathrm{H}_{2} \mathrm{O}$ (Sigma-Aldrich) was then added to yield an equimolar $\mathrm{Mn}^{2+} / \mathrm{DOTA}$ solution. Complete formation of the Mn-DOTA complex was verified with X-band CW-EPR at room temperature. In case of Mims ENDOR experiments, the matrix was deuterated by lyophilisation and redissolution in $\mathrm{D}_{2} \mathrm{O}$. For pulsed EPR measurements, glycerol (or deuterated glycerol) was added as a glassing agent $(50 \%, \mathrm{v} / \mathrm{v})$ to yield a $2 \mathrm{mM} \mathrm{Mn-DOTA}$ solution.

\subsection{Synthesis of ${ }^{13} \mathrm{C}$-DOTA}

${ }^{13} \mathrm{C}$-DOTA was synthesized as reported earlier [30,31]. $0.25 \mathrm{~g}$ Cyclen (1.45 mmol) was dissolved in $2.5 \mathrm{~mL} \mathrm{ddH} \mathrm{H}_{2} \mathrm{O}$. The solution was gently heated to facilitate complete 
dissolution. The alkaline solution was then neutralized with $2 \mathrm{mM}$ hydrochloric acid. In a separate flask, $1 \mathrm{~g}$ of $1,2-{ }^{13} \mathrm{C}$-bromoacetic acid $(7.20 \mathrm{mmol})$ was dissolved in $2.5 \mathrm{~mL}$ $\mathrm{ddH}_{2} \mathrm{O}$. The solution was then cooled with an ice bath and neutralized with sodium hydroxide in such a way that a temperature of $5{ }^{\circ} \mathrm{C}$ was not exceeded. The two solutions were then mixed together and the temperature was elevated to $80^{\circ} \mathrm{C}$. Concentrated sodium hydroxide was added to maintain a $\mathrm{pH}=13$. After the reaction was complete, the solution was acidified to $\mathrm{pH}=2.5$, upon which precipitation occurred. The white precipitate was isolated, washed with ethanol and recrystallized twice in $\mathrm{ddH}_{2} \mathrm{O}$.

${ }^{1} \mathrm{H}$ NMR, $250 \mathrm{MHz}, \mathrm{D}_{2} \mathrm{O}, \delta$ (ppm): 3.78 (s, br, 8H, acetate), 3.28 (s, br, 16H, cyclen ring). MS (ESI+) m/z: $[\mathrm{M}+\mathrm{H}]+$ Calcd for $\mathrm{C} 8{ }^{13} \mathrm{C} 8 \mathrm{H} 28 \mathrm{~N} 4 \mathrm{O} 8$ 413.36; Found 413.24, $[\mathrm{M}+\mathrm{Na}]+$ Calcd for $\mathrm{C} 8{ }^{13} \mathrm{C} 8 \mathrm{H} 28 \mathrm{~N} 4 \mathrm{O} 8$ 435.35; Found 435.24

\subsection{EPR spectroscopy and data analysis}

Pulsed EPR measurements were performed at Q-band on a Bruker E580 spectrometer with a EN 5170 D2 ENDOR probehead. The spectrometer was equipped with either a Bruker AmpQ $10 \mathrm{~W}$ solid state amplifier or a $150 \mathrm{~W}$ traveling-wave tube (Applied Systems Engeneering Inc.) for microwave amplification. EDNMR experiments were performed either in a 'conservative' manner using microwave pulse-forming units and an external SuperQ-FTu ELDOR source for probe and pump frequencies respectively or using a Bruker SpinJet-AWG. In all pulsed experiments the temperature was kept at $5 \mathrm{~K}$ using a continuous-flow helium cryostat (CF935) and an ITC 502 temperature control unit, both from Oxford Instruments.

RF frequencies were generated from a Bruker DICE-II system and amplified with an externally blanked $2 \mathrm{~kW}$ Dressler amplifier (LPA 10020 LF). A 30 MHz low-pass filter (RF Limited DF-3000) was used to cut off higher harmonics. The RF was connected via a Noise Suppressor to the resonator and terminated on the other side with a $50 \Omega$ load.

Echo-detected field swept experiments were performed using the standard Hahn-echo sequence $t_{p} / 2-$ tau $-t_{p}-$ tau echo ( $t_{p}=40 \mathrm{~ns}$, tau $\left.=400 \mathrm{~ns}\right)$. Unless otherwise stated, EDNMR experiments where performed using a tELD $-T-t_{p} / 2-t_{a u}-t_{p}-t_{a u}$ echo sequence with $t_{E L D}=9000 \mathrm{~ns}, \mathrm{~T}=8000 \mathrm{~ns}, \mathrm{t}_{\mathrm{p}}=800 \mathrm{~ns}$, tau $=1800 \mathrm{~ns}$. The integration width of the echo was set to $800 \mathrm{~ns}$, centered around the maximum of the spin echo. 
No transients were collected. The shot repetition time was set to $3000 \mu$ s with 20 to 50 shots per point. The sweep width of the frequency of the ELDOR pulse was $440 \mathrm{MHz}$ with a frequency increment of $0.2 \mathrm{MHz}$, symmetrically placed around the detection frequency. The detection frequency ( $v_{\text {det }}$ ) was chosen to be equal to the frequency of the central dip feature of the resonator bandwidth profile, such that the variation in ELDOR amplitude is approximately symmetric about $v$ det. Amplitudes of ELDOR pulses were extracted from nutation experiments at the detection frequency with subsequent Fourier transformation. Mims ENDOR experiments were performed using the $t_{p}-t_{a u}-t_{p}-t_{R F}-T-t_{p}-$ tau echo pulse sequence with $t_{p}=20 \mathrm{~ns}, t_{R F}=27500 \mu \mathrm{s}$ and $T=5500 \mu \mathrm{s}$. The length of the RF pulse was optimized by nutation experiments of the RF pulse close to v13c. Tau values were incremented in steps of $50 \mathrm{~ns}$ from 300 to $2000 \mathrm{~ns}$. The RF sweep width of $6 \mathrm{MHz}$ started at $9.9 \mathrm{MHz}$ with a frequency increment of $10 \mathrm{kHz}$. The shot repetition time was set to $60 \mathrm{~ms}$ with 1 shot per point. The random acquisition mode was used to minimize baseline artefacts [32]. The ENDOR-efficiency was calculated to $\varepsilon(\tau, \mathrm{LRF})=\left(I_{\mathrm{RF}}-I_{0}\right) / I_{0}$, were $I_{0}$ is an average value of an off-resonant frequency range. The resonator bandwidth for all pulsed EPR experiments was approximately $100 \mathrm{MHz}$.

DFT calculations were performed using the ORCA 4.0 package [33]. The PBE0 hybrid functional with a 6-31+G(d,p) basis set were used for calculation of EPR parameters of the Mn-DOTA complex [34]. The C-PCM solvation model (water as a solvent) was used to obtain more accurate information about aiso [35]. Similar settings were recently used by Un and Bruch in a hyperfine coupling benchmark study of $\mathrm{Mn}^{2+}$ systems [11]. The geometry of the crystal structure of Mn-DOTA was used a starting point for DFT calculations [36].

\section{Results}

The following section is ordered as follows: Section 4.1 deals with the optimization of important experimental parameters in order to obtain Q-band EDNMR spectra with a sufficient narrow width of the central blind spot. Section 4.2 then deals with Q-band EDNMR spectra of different $\mathrm{Mn}^{2+}$ systems, namely the hexaaqua complex, the ${ }^{17} \mathrm{O}-$ labeled hexaaqua complex, Mn-DOTA and $\mathrm{Mn}-{ }^{13} \mathrm{C}$-DOTA. Section 4.3 is dedicated to the investigation and explanation of combination frequencies, which appear when using higher powered ELDOR pulses. Section 4.4 compares EDNMR to 
the Mims ENDOR technique with the aim of getting a better insight into the ${ }^{13} \mathrm{C}$ hyperfine coupling of $\mathrm{Mn}-{ }^{13} \mathrm{C}-\mathrm{DOTA}$.

In terms of nomenclature in this paper; a sq EDNMR transition describes a transition in which the projection of both the electron spin and the nuclear spin is changed $\left(\Delta m_{\mathrm{S}}= \pm 1, \Delta m_{\mathrm{l}}= \pm 1\right)$. Consequently, $\mathrm{dq}$ and tq describe double and triple quantum transitions in which $\Delta m_{s}= \pm 1, \Delta m_{\iota}= \pm 2$ and \pm 3 . Transitions in which two different nuclei change their projection (e.g. ${ }^{1} \mathrm{H}$ and $\left.{ }^{55} \mathrm{Mn}\right)$ are denoted as $\mathrm{mq}$ transitions $\left(\Delta m_{\mathrm{s}}= \pm 1\right.$, $\Delta m_{\mathrm{l}, 1}= \pm 1, \Delta m_{\mathrm{l}, 2}= \pm 1$ (or \pm 2 etc. for high-spin nuclei)) and also called combination frequencies.

\subsection{Optimization of experimental EDNMR parameters}

As already detailed in the previous section, the central blind spot plays a crucial role in EDNMR experiments at Q-band. An important step in setting up a Q-band EDNMR experiment is therefore the optimization of the width of the central blind spot; making it narrow enough so as not to obscure signals from any low- $\gamma$ nuclei present while at the same time using an ELDOR pulse of sufficient power to excite the formally forbidden electron and nuclear transitions. Figure 1 shows a series of EDNMR spectra of $\left[\mathrm{Mn}\left(\mathrm{H}_{2} \mathrm{O}\right)_{6}\right]^{2+}$ with different pulse lengths, amplitudes and shapes for the detection and/or the ELDOR channel. $h$ denotes the hole-depth parameter of the central blind spot, and $\Delta v_{1 / 2}$ the full width at half maximum of the central blind spot. The only nuclei visible are proton signals appearing at ca. $\pm 51 \mathrm{MHz}$. The first three spectra were recorded with 50-100 ns detection pulses and 1000, 4500 and 9000 ns long ELDOR pulses respectively with an identical microwave amplitude $\omega E$ EL. As expected, $h$ and the intensity of the proton signals increases with stronger ELDOR pulses. All three spectra are characterized by an excessive line broadening of the proton signals and the central blind spot caused by relatively unselective detection pulses and a short echo integration window, which is the most important factor in determining the resolution of detectable EDNMR signals [8]. 
rect ELD - 4500/50/100 ns pulses, $\omega_{\text {ELD }}=1.2 \times 10^{7} \mathrm{rad} \mathrm{s}^{-1}$

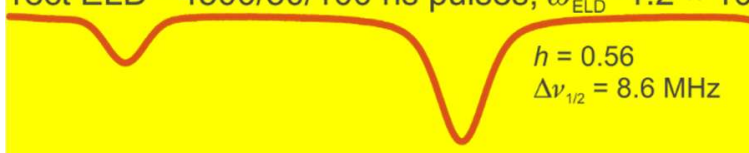

rect ELD - 9000/50/100 ns pulses, $\omega_{\text {ELD }}=1.2 \times 10^{7} \mathrm{rad} \mathrm{s}^{-1}$

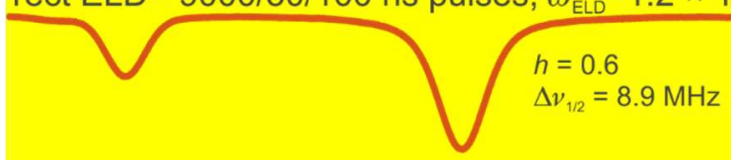

rect ELD $-9000 / 400 / 800 \mathrm{~ns}$ pulses, $\omega_{\text {ELD }}=5.0 \times 10^{7} \mathrm{rad} \mathrm{s}$

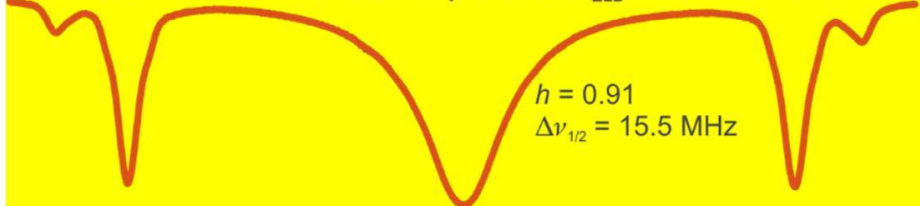

rect ELD - 9000/400/800 ns pulses, $\omega_{\mathrm{ELD}}=2.8 \times 10^{7} \mathrm{rad} \mathrm{s}^{-1}$

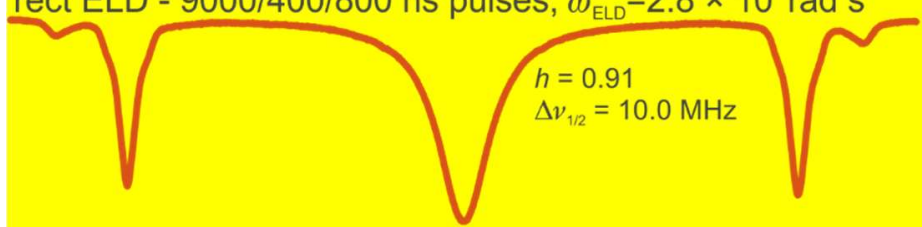

rect ELD $-9000 / 400 / 800 \mathrm{~ns}$ pulses, $\omega_{\mathrm{ELD}}=1.2 \times 10^{7} \mathrm{rad} \mathrm{s}^{-1}$

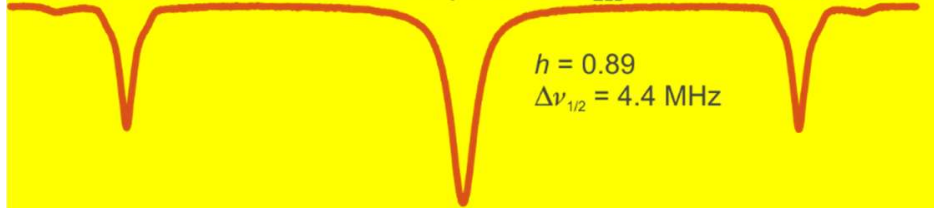

Gaussian ELD - 9000/400/800 ns pulses, $\omega_{\mathrm{ELD}}=1.2 \times 10^{7} \mathrm{rad} \mathrm{s}$

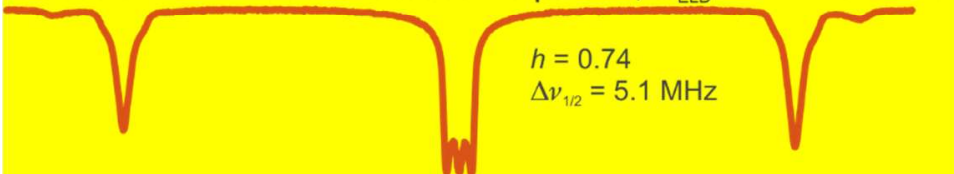

Gaussian ELD - 9000/400/800 ns pulses, $\omega_{\mathrm{ELD}}=5.6 \times 10^{6} \mathrm{rad} \mathrm{s}$

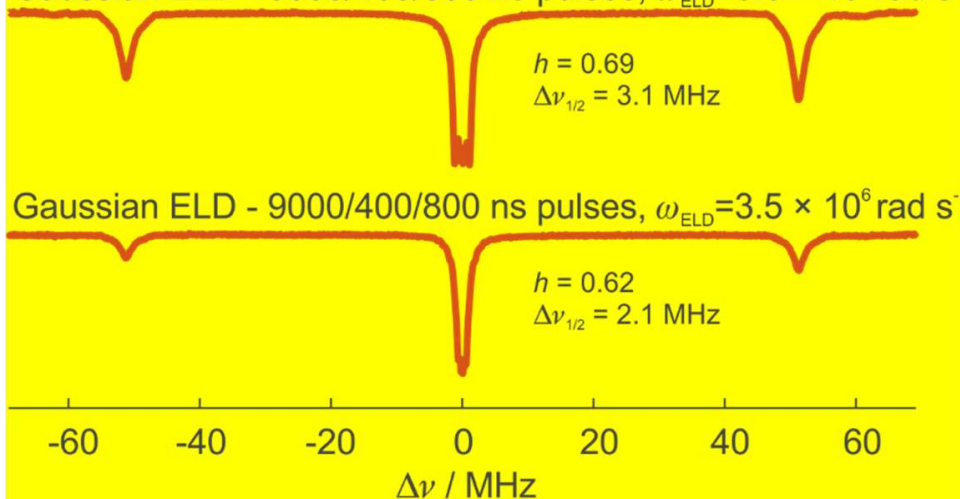

Figure 1: ELDOR-detected NMR spectra of $\left[\mathrm{Mn}\left(\mathrm{H}_{2} \mathrm{O}\right)_{6}\right]^{2+}$ recorded at Q-band frequencies ( $v_{0}=33.855 \mathrm{GHz}$ ) and $5 \mathrm{~K}$ with different pulse lengths, shapes and amplitudes. The magnetic field was selected to be at the position of the $3^{r d}$ hyperfine peak $\left(B_{0}=1206 \mathrm{mT}\right)$. $\omega E L D$ is the amplitude (strength) of the ELDOR pulse, $h$ denotes the holedepth parameter and $\Delta v_{1 / 2}$ the full width at half maximum of the central blind spot. In all cases the strength of the detection pulses was selected to yield a maximum echo intensity. ${ }^{1} \mathrm{H}$ signals are centered around $\left| \pm v_{1 H}\right|=51.3$ $\mathrm{MHz}$. For more experimental details see Section 3.3. 
The $4^{\text {th }}, 5^{\text {th }}$ and $6^{\text {th }}$ spectra were recorded with highly selective $400-800$ ns detection pulses, and $9000 \mathrm{~ns}$ long ELDOR pulses with different microwave amplitudes. With strong ELDOR pulses $\left(\omega_{E L D}=5.0 \times 10^{7} \mathrm{rad} \mathrm{s}^{-1}\right)$ an almost-complete saturation of the allowed EPR transition as well as the forbidden proton transition is possible. Although spectra recorded with highly powered ELDOR pulses are characterized by a broad central blind spot, which renders detection of low- $\gamma$ nuclei difficult, the line shape of proton signals is much better resolved in comparison to the first three spectra. The proton line shape did not change when using ELDOR pulses of shorter length than 9000 ns with $400-800$ ns detection pulses, indicating that any magnetization transfer artefact from the $9000 \mathrm{~ns}$ long ELDOR pulse can be neglected [8].

The signals appearing at approximately $\pm 60 \mathrm{MHz}$ in the $4^{\text {th }}$ and $5^{\text {th }}$ spectra are part of ${ }^{55} \mathrm{Mn}-{ }^{1} \mathrm{H}$ combination frequencies, which are discussed in detail in Section 4.4.

The influence of the length of the detection pulses and the echo integration window on EDNMR is best visible when comparing the $3^{\text {rd }}$ spectrum to the $6^{\text {th }}$ spectrum in which all experimental settings are identical except for the length of the detection pulses and the echo integration window; the shorter detection pulses and integration window leads to a broadening in the detected proton signals.

Consequently, Q-band EDNMR is always a compromise between the signal-to-noise ratio and the width of the central blind spot. For our Q-band studies, the most convenient setup consisted of a $9000-400-800 \mathrm{~ns}$ pulse scheme with $\omega E L D=1.2 \times 10^{7} \mathrm{rad} \mathrm{s}^{-1}$. In case of low concentrations (e.g. membrane proteins), it may be better to use 200-400 ns detection pulses, as the line broadening of the proton signals and the central blind spot was seen to be minimal in our studies. The shape of the central blind spot for rectangular ELDOR pulses was a pure Lorentzian as expected [17], with minor deviations stemming from a non-symmetric resonator profile.

As previously demonstrated in the original hole-burning EPR techniques by the group of Arthur Schweiger (FT-detected NMR and ELDOR-detected NMR) [1,37], the use of Gaussian shaped ELDOR-pulses leads to a considerable narrowing of the central blind spot, especially at its flanks, while retaining the intensity of the forbidden proton transitions ( $6^{\text {th }}$ and $7^{\text {th }}$ spectrum in Figure 1). The FWHM of the central blind spot can be reduced even further ( $8^{\text {th }}$ and $9^{\text {th }}$ spectrum in Figure 1 ) by reducing the power of the ELDOR pulse. For $\omega_{E L D}=3.5 \times 10^{6} \mathrm{rad} \mathrm{s}^{-1}$, however, the intensities of the forbidden transitions become rather small, especially as proton signals typically give the most 
intense EDNMR signals. The 'trident'-like feature close to $v^{0}$, which is visible in the $7^{\text {th }}$, $8^{\text {th }}$ and partially in the $9^{\text {th }}$ spectrum of Figure 1 , must not be mistaken as a real signal. It is rather an artefact stemming from an imperfect Gaussian line shape of the ELDOR pulse. These artifacts can be distinguished from possible real signals by reducing the ELDOR amplitude and carefully monitoring the frequency of the observed signal. Upon reducing the ELDOR amplitude from $\omega E L D=1.2 \times 10^{7} \mathrm{rad} \mathrm{s}^{-1}$ to $\omega_{E L D}=5.9 \times 10^{6} \mathrm{rad} \mathrm{s}^{-1}$ the outer 'triplet signal' maxima move from $\pm 1.9 \mathrm{MHz}$ to \pm 1.1 $\mathrm{MHz}\left(7^{\text {th }}\right.$ and $8^{\text {th }}$ spectrum in Figure 1). If the 'triplet signal' would be real, one would expect a slight increase in frequency (if any), as at lower ELDOR powers the signals will convolute less with the narrower blind spot function. The use of Gaussian shaped detection pulses did not yield improved EDNMR spectra in terms of the FWHM of the central blind spot in comparison to rectangular detection pulses.

\subsection{Q-band EDNMR spectra of $\mathrm{Mn}^{2+}$ complexes}

Figure 2 shows a series of $\mathrm{Q}$-band EDNMR spectra of different $\mathrm{Mn}^{2+}$ complexes; namely the hexaaqua complex $\left(\left[\mathrm{Mn}\left(\mathrm{H}_{2} \mathrm{O}\right)_{6}\right]^{2+}\right)$, the ${ }^{17} \mathrm{O}$-labeled hexaaqua complex $\left(\left[\mathrm{Mn}\left(\mathrm{H}_{2}{ }^{17} \mathrm{O}\right)_{6}\right]^{2+}\right)$, the Mn-DOTA complex and the ${ }^{13} \mathrm{C}$-labeled Mn-DOTA complex. The echo-detected field-swept spectra (EDFS) of all four compounds are shown as insets on the left hand side of each EDNMR spectrum with asterisks denoting the magnetic field positions for EDNMR experiments. Not surprisingly, the ${ }^{17}$ O-labeled hexaaqua shows the broadest EDFS spectrum due to unresolved ${ }^{17} \mathrm{O}$ hyperfine couplings with $\mathrm{FWHM}=2.4 \mathrm{mT}$ for the $3^{\text {rd }}$ hyperfine peak, whereas $\mathrm{FWHM}=1.6 \mathrm{mT}$ for the unlabeled hexaaqua complex. The EDFS of Mn-DOTA complexes are characterized by a smaller FWHM of $0.9 \mathrm{mT}$, which is expected, as the Mn-DOTA complexes have a smaller zerofield splitting compared to the hexaaqua complex [38].

The EDNMR spectra of the unlabeled hexaaqua complex were recorded at three different magnetic field positions ( $1^{\text {st }}, 3^{\text {rd }}$ and $6^{\text {th }}$ hyperfine peak). Signals of the strongly coupled ${ }^{55} \mathrm{Mn}$ nucleus ( ${ }^{555 \mathrm{Mn}}=12.7 \mathrm{MHz}, I=5 / 2$ ) appear at approximately $\pm 155 \mathrm{MHz}$ and $\pm 115 \mathrm{MHz}$ respectively. As expected, the two signals at the right hand side and the left hand side of the EDNMR spectrum are centered at $\left|a_{\text {iso }}\right| / 2$, which yields an isotropic ${ }^{55} \mathrm{Mn}$ hyperfine coupling of $\left|a_{\text {iso }}\right|=269 \mathrm{MHz}$. It should be noted that the isotropic hyperfine coupling of ${ }^{55} \mathrm{Mn}$ is known to be negative [39]. Throughout this article, absolute values are given for the hyperfine coupling as EDNMR spectra are not sensitive to the sign of the isotropic hyperfine coupling. If the magnetic field is set to 
one of the outer hyperfine peaks (yellow $/ 1^{\text {st }}$ or blue/2 ${ }^{\text {nd }}$ EDNMR spectra in Figure 2), the EDNMR spectra become asymmetric with respect to the ${ }^{55} \mathrm{Mn}$ resonances. For outer hyperfine transitions, each allowed EPR transition $\left(\Delta m_{\mathrm{s}}= \pm 1, m_{\mathrm{l}}=-5 / 2\right.$ or $\Delta m_{\mathrm{s}}= \pm 1, m_{\mathrm{l}}=+5 / 2$, yellow $/ 1^{\text {st }}$ and blue $/ 2^{\text {nd }}$ spectra in Figure 2 respectively) is connected to 2 forbidden EDNMR transitions only. If the magnetic field is on resonant with one of the four inner hyperfine peaks, each allowed EPR transition is connected to four forbidden ${ }^{55} \mathrm{Mn}$ EDNMR transitions (red/3rd spectrum in Figure 2). For a distinct labeling of the different forbidden transitions, the reader is referred to Figure 2.

The splitting between the two ${ }^{55} \mathrm{Mn}$ resonances on each side of the EDNMR spectra is larger than the expected first order expression of $\Delta v=2 v 55 \mathrm{Mn}=26 \mathrm{MHz}$ for a strongly coupled ${ }^{55} \mathrm{Mn}$ nucleus. Sturgeon et al. used perturbation up to the third order to evaluate the splitting in their ${ }^{55} \mathrm{Mn} \mathrm{X}$-band ENDOR spectra [40]. As proposed in their study, the deviation of the splitting from $\Delta v=2 v_{55} \mathrm{Mn}$ decreases with higher microwave frequencies from $70 \%$ at Q-band ( $\Delta v=44 \mathrm{MHz}$, as observed in this study) to $10 \%$ at W-band ( $\Delta v=77 \mathrm{MHz}$ where $\Delta v=2 v 55 \mathrm{Mn}=70 \mathrm{MHz}$, Cox et al. [5]). In addition, the quadrupole interaction of the ${ }^{55} \mathrm{Mn}$ nucleus is also known to affect the position of the ${ }^{55} \mathrm{Mn}$ lines slightly [41].

In contrast to strongly coupled ${ }^{55} \mathrm{Mn}$ nuclei, weakly coupled ${ }^{1} \mathrm{H}$ nuclei, observed as signals at ca. $\pm 51 \mathrm{MHz}$, are not subject to asymmetries depending on the magnetic field positions. This is because the hyperfine coupling of weakly coupled ${ }^{1} \mathrm{H}$ nuclei is smaller than the inhomogeneous EPR linewidth. As a consequence, the detection pulses excite both allowed EPR transitions simultaneously (e.g. $\Delta m_{\mathrm{s}}= \pm 1, m_{\mathrm{l}}=-1 / 2$ and $\Delta m s= \pm 1, m_{\mathrm{I}}=+1 / 2$ ), thus making the ${ }^{1} \mathrm{H}$ EDNMR spectrum symmetric around the central blind spot at all field positions.

The EDNMR spectrum of the ${ }^{17} \mathrm{O}-$ labeled hexaaqua complex in Figure 2 (purple/4 $4^{\text {th }}$ spectrum) shows a more complex spectrum than the hexaaqua complex. Although the resonance frequencies of the ${ }^{55} \mathrm{Mn}$ sq EDNMR transitions at $\pm 155 \mathrm{MHz}$ and $\pm 115 \mathrm{MHz}$ remain unchanged, many satellite transitions appear on both sides of the spectrum at the expense of ${ }^{55} \mathrm{Mn}$ sq signal intensity. A more detailed explanation is given in Section 4.4 of this article. Additional signals close to the blind spot region belong to resonances from the ${ }^{17} \mathrm{O}$ nucleus $\left(v_{170}=6.9 \mathrm{MHz}, I=5 / 2\right.$ ). The advantage of using a Gaussian shaped ELDOR pulse over a rectangular ELDOR pulse is illustrated in the inset, showing a close-up of the blind spot region of the ${ }^{17} \mathrm{O}-$ labeled hexaaqua complex. An 
intense signal close to $v_{170}$ at $6.3 \mathrm{MHz}$, originating from $2^{\text {nd }}$ shell ${ }^{17} \mathrm{O}$ nuclei, is visible in both cases. The deviation from $v_{170}$ is most likely caused by a convolution with the function of the central blind spot. The signal at $9.9 \mathrm{MHz}$ originates from $1^{\text {st }}$ coordination sphere ${ }^{17} \mathrm{O}$ nuclei in the $m s=-1 / 2$ manifold. The splitting to the matrix line is $3.7 \mathrm{MHz}$, which is half the ${ }^{17} \mathrm{O}$ hyperfine coupling reported in previous studies ( $\mid$ aiso $\mid=7.5 \mathrm{MHz}$ ) $[5,42,43]$. The EDNMR signals of $1^{\text {st }}$ coordination sphere ${ }^{17} \mathrm{O}$ nuclei in the $m_{\mathrm{s}}=+1 / 2$ manifold are expected at approximately $2.5 \mathrm{MHz}$ and are not resolved due to severe overlap with the blind spot. Although a 'signal' is visible at $2.3 \mathrm{MHz}$ for Gaussian shaped ELDOR pulses, it is not possible to conclusively say if this is an actual signal of the ${ }^{17} \mathrm{O}$ nuclei or rather an artefact caused by Gaussian shaped ELDOR pulses, which is also visible in Figure 1. The signals in the range around $20 \mathrm{MHz}$ are difficult to analyze with rectangular ELDOR pulses. With Gaussian shaped ELDOR pulses it is clear however that two signals can be clearly distinguished. The first signal resonates at $17.3 \mathrm{MHz}$ and stems from ${ }^{17} \mathrm{O}$ nuclear transitions in the $m_{\mathrm{s}}=-3 / 2$ manifold. The signal at $20.3 \mathrm{MHz}$ is assigned to a ${ }^{17} \mathrm{O}$ nuclear double quantum transition $\left(\Delta m_{\mathrm{I}}= \pm 2\right)$. To first order, double quantum resonances are centered at $2 v_{170}$ and split by $2 A_{170}$. The second double quantum signal is expected at approximately $7 \mathrm{MHz}$ and therefore is most likely obscured by the more intense sq signals. The broad signal at $31.0 \mathrm{MHz}$ is finally assigned to $a{ }^{17} \mathrm{O}$ nuclear triple quantum transition $\left(\Delta m_{\mathrm{I}}= \pm 3\right)$. Here, signals are expected to be centered at $3 v 170$ and split by $3 A_{170}$.

The surprisingly large intensity of nuclear double and even triple quantum transitions can be explained by evaluating the different contributions to the spin Hamiltonian. Firstly, ${ }^{17} \mathrm{O}$ is a high-spin nuclei with a relatively large quadrupole interaction of $e^{2} q Q / h=7 \mathrm{MHz}$ and $\eta=1$ [43]. This leads to larger off-diagonal elements in the spin Hamiltonian and therefore an increased level mixing of nuclear spin states and an increased transitions probability, $l_{\text {f. }}$ This effect is increased at $Q$-band frequencies, as the quadrupole interaction, the principal values of the hyperfine tensor and the nuclear Zeeman interaction have very similar magnitudes $[44,45]$. 


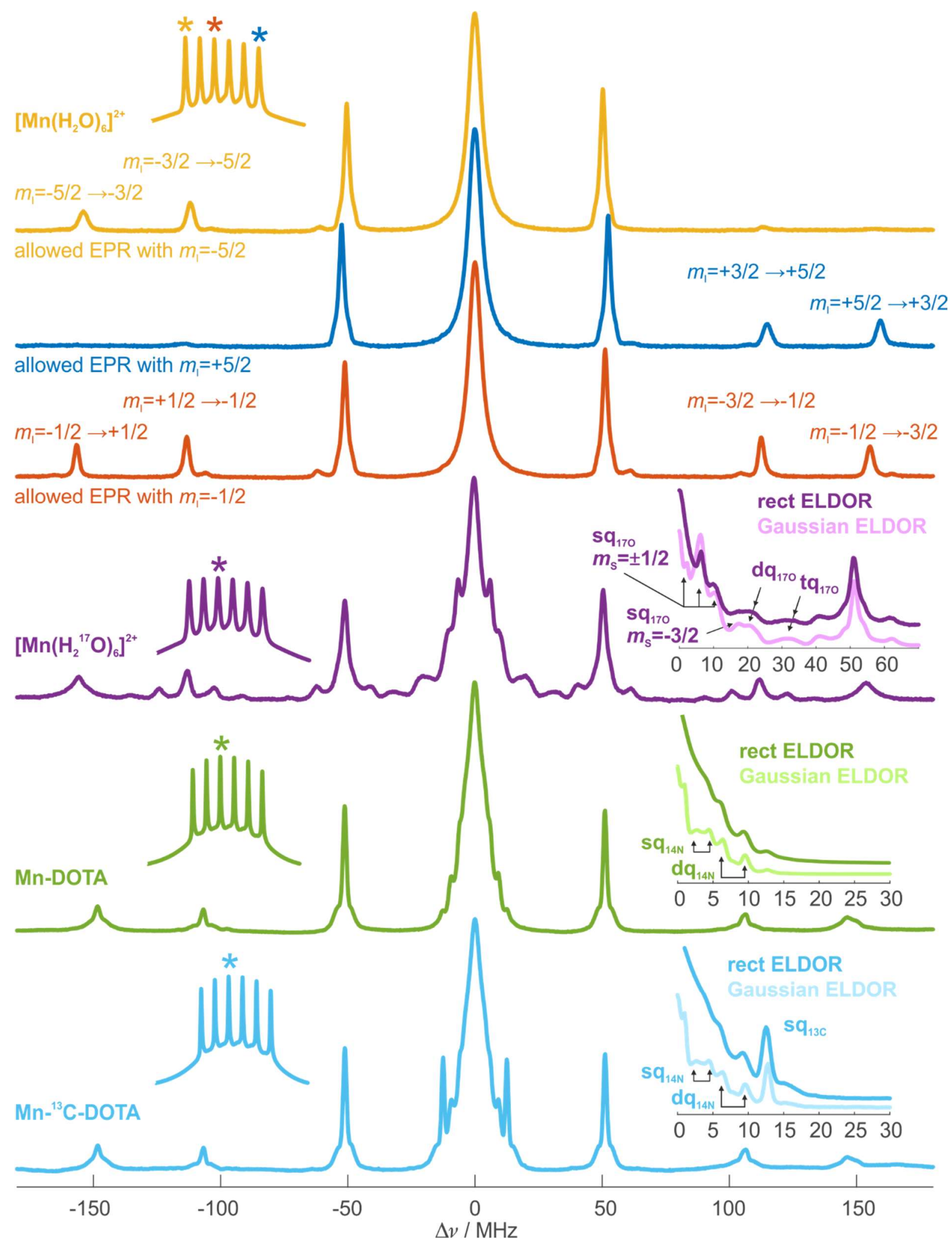

Figure 2: Spectra from the top of figure: Q-band EDNMR of $\left[\mathrm{Mn}\left(\mathrm{H}_{2} \mathrm{O}\right)_{6}\right]^{2+}$ at different magnetic fields $\left(1^{\text {st }}, 3^{\text {rd }}\right.$ and $6^{\text {th }}$ hyperfine peak), $\left[\mathrm{Mn}\left(\mathrm{H}_{2}{ }^{17} \mathrm{O}\right)_{6}\right]^{2+}, \mathrm{Mn}-\mathrm{DOTA}$ and $\mathrm{Mn}-{ }^{13} \mathrm{C}-\mathrm{DOTA}$ ( $3^{\text {rd }}$ hyperfine peak). Spectra were normalized to $[0,1]$ and inverted. Echo detected field-swept spectra are shown as insets on the left and expansions of the blind spot region with rectangular $\left(\omega E L D=1.2 \times 10^{7} \mathrm{rad} \mathrm{s}^{-1}\right)$ or Gaussian shaped ELDOR pulses $\left(\omega_{E L D}=6.0 \times 10^{6} \mathrm{rad} \mathrm{s} \mathrm{s}^{-1}\right.$, pale colour) are show as insets on the right. For the hexaaqua complex, forbidden transitions are labeled for different allowed EPR transitions ( $\Delta m_{S}= \pm 1$ is omitted for sake of clarity). Strongly coupled ${ }^{55} \mathrm{Mn}$ signals appear around \pm 150 and $\pm 110 \mathrm{MHz}$, whereas weakly coupled protons are centered around $\left| \pm v_{1 H}\right|=51 \mathrm{MHz}$. Larmor frequencies of other relevant nuclei: $v 170=6.9 \mathrm{MHz}, I=5 / 2 ; v_{14 \mathrm{~N}}=3.7 \mathrm{MHz}, I=1 ; v_{13 \mathrm{C}}=12.8 \mathrm{MHz}, I=1 / 2$. 
The last two spectra show Q-band EDNMR spectra of a Mn-DOTA complex, either unlabeled or partially ${ }^{13} \mathrm{C}$-labeled (see Section 3.2 for more experimental details). Due to the smaller ${ }^{55} \mathrm{Mn}$ hyperfine coupling of $\left|a_{\text {iso }}\right|=255 \mathrm{MHz}$ of $\mathrm{Mn}-\mathrm{DOTA}$, the ${ }^{55} \mathrm{Mn}$ resonances are centered around a slighter smaller frequency value. The signals at $\pm 150 \mathrm{MHz}$ and $\pm 110 \mathrm{MHz}$ are broader than the ${ }^{55} \mathrm{Mn}$ signals of the hexaaqua complex, which is due to unresolved ${ }^{55} \mathrm{Mn}-{ }^{14} \mathrm{~N}$ combination signals (see Section 4.3). The width of the proton signals of $\mathrm{Mn}$-DOTA at $\pm 51 \mathrm{MHz}$ are clearly smaller than the proton signals of the hexaaqua complex, which is in agreement with ${ }^{1} \mathrm{H}-\mathrm{ENDOR}$ [28]. The insets reveal many signals close to the blind spot region for both complexes. The signals at 2.7 MHz and 4.4 MHz have been identified as sq EDNMR transitions of the ${ }^{14} \mathrm{~N}$ nucleus $(\nu 14 \mathrm{~N}=3.7 \mathrm{MHz}, I=1)$, as they are centered around the $114 \mathrm{~N}$ Larmor frequency and split by $1.7 \mathrm{MHz}$, which is similar to reported values for $A_{14 \mathrm{~N}}$ in $\mathrm{Mn}$ DOTA systems [28,38]. The signals appearing at 6.4 and $9.6 \mathrm{MHz}$ are consequently assigned to ${ }^{14} \mathrm{~N}$ dq EDNMR transitions. The peak at $12.8 \mathrm{MHz}$ belongs to a sq transition of ${ }^{13} \mathrm{C}$ nuclei $\left(v_{13 \mathrm{C}}=12.8 \mathrm{MHz}, I=1 / 2\right)$. Interestingly, a signal with a much smaller intensity is also visible in the unlabeled sample at the same frequency. We assign this feature to natural abundance ${ }^{13} \mathrm{C}$, which is plausible as firstly EDNMR is known to be sensitive enough to detect natural abundance ${ }^{13} \mathrm{C}$ [10], and secondly Mn-DOTA offers a very carbon rich environment. Q-band EDNMR was not able to resolve the rather large and recently reported ${ }^{14} \mathrm{~N}$ quadrupole interaction $\left(e^{2} q Q / h=4.5 \mathrm{MHz}\right)$ [28]. Just as in the case of ${ }^{17} \mathrm{O}$, the ${ }^{14} \mathrm{~N}$ quadrupole interaction is of comparable magnitude to the nuclear Zeeman interaction and the hyperfine interaction leading to strong mixing of nuclear spin states, which results in an increased forbidden transition probability, If.

\subsection{Combination frequencies}

Some nuclei exhibit a relatively small forbidden transition probability, $I_{\mathrm{f}}{ }^{31} \mathrm{P}$ $\left(v_{31 \mathrm{P}}=20.8 \mathrm{MHz}, I=1 / 2\right)$ for example, is a spin-1/2 nuclei with a reasonably large isotropic hyperfine coupling constant and a small dipolar hyperfine contribution for weakly coupled ligand ${ }^{31} \mathrm{P}$ nuclei in $\mathrm{Mn}^{2+}$ systems [11,27]. Both features lead to a smaller $I_{f}$, which is disadvantageous for detecting these interactions using EDNMR. In some cases, especially at lower concentrations or in cases of poor ligand binding, it is therefore useful to use higher-powered ELDOR pulses to increase the intensity of the signals observed. Higher-powered ELDOR pulses, however, also lead to the 
appearance of combination frequencies involving the simultaneous excitation of two different nuclei $\left(\Delta m_{\mathrm{S}}= \pm 1, \Delta m_{\mathrm{l}, 1}= \pm 1, \Delta m_{\mathrm{l}, 2}= \pm 1\right)$. Combination frequencies have previously been observed at W-band frequencies [5,8]. At Q-band, combination frequencies will be intrinsically more intense, as If $\alpha 1 / v^{2}$. In addition, more weakly coupled nuclear will approach the 'cancellation condition'; $A_{l}=2$ и. In this section we therefore include a more in-depth explanation of combination frequencies at Q-band, with reference to the systems presented in Figure 2.

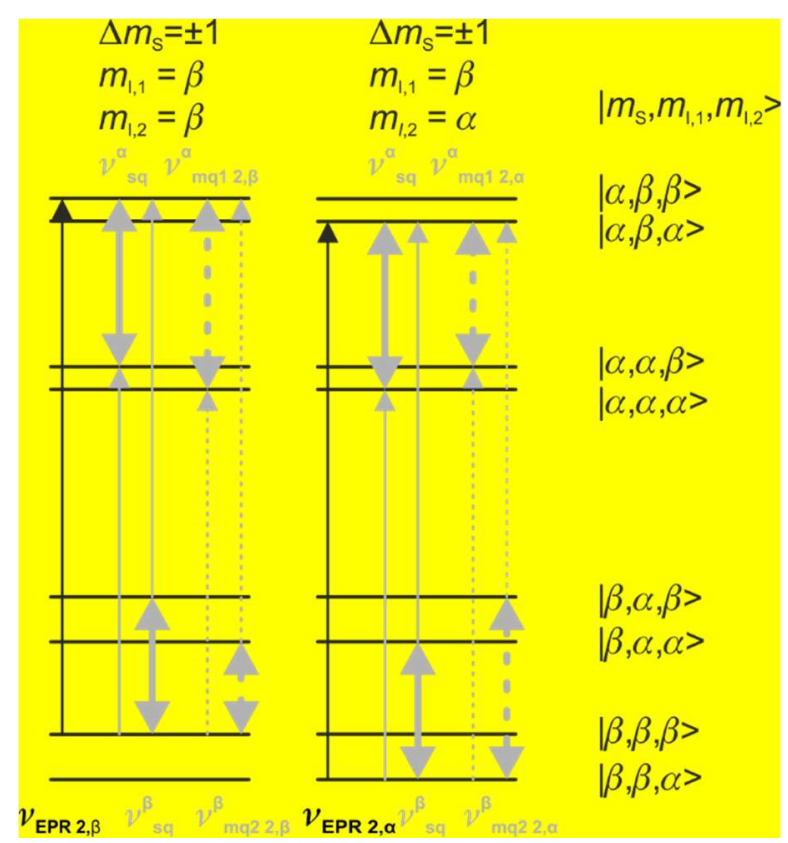

Figure 3: Spin-ladder energy diagram of $S=1 / 2$ coupled to two nuclei with $I_{1}=1 / 2$ and $I_{2}=1 / 2$. The first nuclei is strongly coupled $\left(A_{1}<0\right.$ and $\left.\gamma_{N, 1}>0\right)$. As a consequence, a selective excitation of the allowed EPR transition with $\Delta m_{S}= \pm 1, \Delta m_{l, 1}=\beta$ is possible. The second nuclei is weakly coupled with $A_{2}>0$ and $\gamma_{N, 2}>0$. Therefore, both allowed EPR transitions are excited simultaneously. Black lines: allowed EPR transitions with either $m_{l, 1}=\beta$, $m_{l, 2}=\beta$ (left) or $m_{l, 1}=\beta, m_{l, 2}=\alpha$ (right). Solid grey lines: EDNMR sq transitions with $\Delta m_{s}= \pm 1, \Delta m_{l, 1}= \pm 1$ and $\Delta m_{l, 2}=0$. Dotted grey lines: EDNMR mq transitions with $\Delta m_{S}= \pm 1, \Delta m_{l, 1}= \pm 1$ and $\Delta m_{l, 2}= \pm 1$. For all types of lines: Thin lines correspond to electronic transition frequencies (ca. $34 \mathrm{GHz}$ at Q-band) whereas bold lines correspond to nuclear frequencies $(\mathrm{MHz})$.

Figure 3 shows a spin-ladder diagram of an unpaired electron with $S=1 / 2$ coupled to two nuclei with $I_{1}=1 / 2$ and $I_{2}=1 / 2$. In the diagram the first nuclei is strongly coupled with $A_{1}<0$ and $2 \mathrm{~N}, 1>0$, in the same way that we have already seen for the central nuclear transition of ${ }^{55} \mathrm{Mn}$. The second nucleus is weakly coupled with $A_{2}>0$ and $2 \mathrm{~N}, 2>0$, as observed for a proton coupled to $\mathrm{Mn}^{2+}$ in all of the systems shown in Figure 2. In such a case the spin Hamiltonian is defined as:

$$
\mathcal{H}=\beta_{e} \boldsymbol{B g} \boldsymbol{S} / h-\beta_{N, 1} g_{N, 1} \boldsymbol{B S} / h+\boldsymbol{S} \boldsymbol{A}_{1} \boldsymbol{I}_{1}-\beta_{N, 2} g_{N, 2} \boldsymbol{B S} / h+\boldsymbol{S A}_{2} \boldsymbol{I}_{2}
$$

Assuming the high field approximation is valid for electron and nuclear Zeeman interactions, the frequencies of the allowed EPR transitions are given by: 


$$
\begin{aligned}
& v_{E P R \mid 2, \beta}=E_{\alpha \beta \beta}-E_{\beta \beta \beta}=v_{S}+A_{1} / 2-A_{2} / 2 \\
& v_{E P R \mid 2, \alpha}=E_{\alpha \beta \alpha}-E_{\beta \beta \alpha}=v_{S}+A_{1} / 2+A_{2} / 2
\end{aligned}
$$

vs describes the Larmor frequency of the electron. In accordance with Figure 3 two allowed EPR transitions are found, as for the strongly coupled nucleus $/ 1$ only the allowed EPR transition with $m_{1,1}=\beta$ is considered. In the following text, subscripted expressions describe the 'type' of transitions (EPR, sq, or mq) and to which allowed EPR transitions they are connected $\left(m_{1,2}=\beta\right.$ or $m_{1,2}=\alpha$, see Figure 3$)$. Superscripted expressions describe the electron spin manifold from which the nuclear frequencies stem. For forbidden transitions of the type $\Delta m_{\mathrm{s}}= \pm 1, \Delta m_{\mathrm{l}, 1}= \pm 1$ one finds:

$$
\begin{aligned}
& v_{s q 1 \mid 2, \beta}=E_{\alpha \alpha \beta}-E_{\beta \beta \beta}=v_{S}-v_{I, 1}-A_{2} / 2 \\
& v_{s q 2 \mid 2, \beta}=E_{\alpha \beta \beta}-E_{\beta \alpha \beta}=v_{S}+v_{I, 1}-A_{2} / 2 \\
& v_{s q 1 \mid 2, \alpha}=E_{\alpha \alpha \alpha}-E_{\beta \beta \alpha}=v_{S}-v_{I, 1}+A_{2} / 2 \\
& v_{s q 2 \mid 2, \alpha}=E_{\alpha \beta \alpha}-E_{\beta \alpha \alpha}=v_{S}+v_{\mathrm{I}, 1}+A_{2} / 2
\end{aligned}
$$

Forbidden transitions of the type $\Delta m_{\mathrm{S}}= \pm 1, \Delta m_{\mathrm{l}, 1}= \pm 1, \Delta m_{\mathrm{l}, 2}= \pm 1$ yield:

$$
\begin{aligned}
& v_{m q 1 \mid 2, \beta}=E_{\alpha \alpha \alpha}-E_{\beta \beta \beta}=v_{S}-v_{I, 1}-v_{I, 2} \\
& v_{m q 2 \mid 2, \beta}=E_{\alpha \beta \beta}-E_{\beta \alpha \alpha}=v_{S}+v_{I, 1}+v_{I, 2} \\
& v_{m q 1 \mid 2, \alpha}=E_{\alpha \alpha \beta}-E_{\beta \beta \alpha}=v_{S}-v_{I, 1}+v_{I, 2} \\
& v_{m q 2 \mid 2, \alpha}=E_{\alpha \beta \alpha}-E_{\beta \alpha \beta}=v_{S}+v_{I, 1}-v_{I, 2}
\end{aligned}
$$

To find the nuclear/EDNMR frequencies, the frequencies of the corresponding allowed EPR transitions must be subtracted from the different forbidden transitions defined above:

$$
\begin{gathered}
v_{s q}^{\alpha}=v_{s q 1 \mid 2, \beta}-v_{E P R \mid 2, \beta}=v_{s q 1 \mid 2, \alpha}-v_{E P R \mid 2, \alpha}=-v_{I, 1}-A_{1} / 2 \\
v_{s q}^{\beta}=v_{s q 2 \mid 2, \beta}-v_{E P R \mid 2, \beta}=v_{s q 2 \mid 2, \alpha}-v_{E P R \mid 2, \alpha}=v_{I, 1}-A_{1} / 2
\end{gathered}
$$


These frequencies reflect the familiar strong coupling regime. However, in the case of $\mathrm{Mn}^{2+}$ these expressions are not valid as the large hyperfine coupling and the high-spin system mandate the use of higher order terms [40]. Nevertheless, they can be used to gain a more complete understanding of the combination peaks. The EDNMR frequencies of the combination peaks can be calculated by:

$$
\begin{aligned}
v_{m q \mid 2, \beta}^{\alpha}=v_{m q \mid 2, \beta}-v_{E P R \mid 2, \beta} & =-v_{I I, 1}-A_{1} / 2-v_{I, 2}+A_{2} / 2 \\
& =v_{s q}^{\alpha}-v_{I, 2}+A_{2} / 2 \\
v_{m q \mid 2, \beta}^{\beta}=v_{m q \mid 2, \beta}-v_{E P R \mid 2, \beta} & =v_{I, 1}-A_{1} / 2+v_{I, 2}+A_{2} / 2 \\
& =v_{s q}^{\beta}+v_{I, 2}+A_{2} / 2 \\
v_{m q \mid 2, \alpha}^{\alpha}=v_{m q \mid 2, \alpha}-v_{E P R \mid 2, \alpha} & =-v_{I, 1}-A_{1} / 2+v_{I, 2}-A_{2} / 2 \\
& =v_{s q}^{\alpha}+v_{I, 2}-A_{2} / 2 \\
v_{m q 2 \mid 2, \alpha}^{\beta}=v_{m q 2 \mid 2, \alpha}-v_{E P R \mid 2, \alpha} & =v_{I, 1}-A_{1} / 2-v_{I, 2}-A_{2} / 2 \\
& =v_{s q}^{\beta}-v_{I, 2}-A_{2} / 2
\end{aligned}
$$

Equations 1.19-1.22 show that two combination frequencies are centered around each sq EDNMR transition, although it should be noted that these two combination frequencies are connected to different allowed EPR transitions (VEPR $2, \alpha$ or $\operatorname{VEPR} \mid 2, \beta$ ). The combination frequencies of Equation 1.19 and 1.21 are centered around the sq EDNMR transition $\nu_{\text {sq }}^{\alpha}$ and split by $2 \eta_{, 2}-A_{2}$. Combination frequencies of Equation 1.20 and 1.22 are centered around $\nu_{\mathrm{sq}}$ and split by $2 u, 2+A_{2}$ :

$$
\begin{gathered}
\Delta v_{111, I 2}=v_{m q 2 \mid 2, \beta}^{\beta}-v_{m q 2 \mid 2, \alpha}^{\beta}=2 v_{I, 2}+A_{2} \\
\Delta v_{211, I 2}=v_{m q 1 \mid 2, \alpha}^{\alpha}-v_{m q 1 \mid 2, \beta}^{\alpha}=2 v_{I, 2}-A_{2}
\end{gathered}
$$

Figure 4 shows a series of $\mathrm{Mn}^{2+}$ Q-band EDNMR spectra recorded using higher powered ELDOR pulses to allow the combination frequencies of the type $\Delta m_{\mathrm{S}}= \pm 1$, $\Delta m_{\mathrm{l}, 1}= \pm 1, \Delta m_{\mathrm{l}, 2}= \pm 1$ or even $\Delta m_{\mathrm{s}}= \pm 1, \Delta m_{\mathrm{l}, 1}= \pm 1, \Delta m_{\mathrm{l}, 2}= \pm 2$ to be resolved. Only the left-hand side with the magnetic field set to the $3^{\text {rd }}$ hyperfine peak is shown; here the $\Delta m_{\mathrm{S}}= \pm 1, m_{55 \mathrm{Mn}}=-1 / 2 \leftrightarrow+1 / 2$ transitions are excited, which exhibit a narrower linewidth than the outer ${ }^{55} \mathrm{Mn}$ nuclear spin manifolds $[25,26]$. For the unlabeled hexaaqua complex, four distinct additional signals are visible which correspond to the 
four combination frequencies given in Equations $1.19-1.22$ and which are drawn in Figure 3. The signals marked with $\Delta v 1,1 \mathrm{H} 55 \mathrm{Mn}$ are centered almost perfectly around the ${ }^{55} \mathrm{Mn}$ sq transition at $-113.3 \mathrm{MHz}$ (the experimentally measured center between the signals was: $\tilde{v} 1,1 \mathrm{H} 55 \mathrm{Mn}=113.4 \mathrm{MHz}$, with a frequency separation between the peaks of: $\Delta v 1,1 \mathrm{H} 55 \mathrm{Mn}=103.0 \mathrm{MHz}$ ). From this point on $\tilde{v}$ notates the experimentally measured center frequency between two peaks and $\Delta v$ the frequency separation (splitting) between them. The signals marked with $\Delta v_{2}, 1{ }_{1} 55 \mathrm{Mn}$, on the other hand, are centered around the ${ }^{55} \mathrm{Mn}$ sq transition at $-156.6 \mathrm{MHz} \quad(\tilde{v} 2,1 \mathrm{H} 55 \mathrm{Mn}=-156.6 \mathrm{MHz}$, $\Delta v 2,1 \mathrm{H} 55 \mathrm{Mn}=101.6 \mathrm{MHz})$.

As predicted by Equations $1.19-1.22$, the splitting of $\Delta v 1,1 \mathrm{H} 55 \mathrm{Mn}$ is slightly larger, which is due to additive hyperfine coupling. The difference of $c a$. $2 \mathrm{MHz}$ between $\Delta v 1,1 \mathrm{H} 55 \mathrm{Mn}$ and $\Delta v_{2}, 1{ }_{1 H 55 \mathrm{Mn}}$ is not an error of the experiment. Indeed, it was reproduced with different samples at high accuracy. ${ }^{1} \mathrm{H}-{ }^{55} \mathrm{Mn}$ combination frequencies are also visible in all other samples, although they shift to smaller frequencies for Mn-DOTA complexes, as here $\left|a_{\text {iso }}\right|$ gets smaller and correspondingly the ${ }^{55} \mathrm{Mn}$ sq peaks shift to smaller absolute frequencies $(-148.3 \mathrm{MHz}$ and $-106.7 \mathrm{MHz})$. For Mn-DOTA complexes it was found that: $\tilde{v} 1,1 \mathrm{H} 55 \mathrm{Mn}=-106.0 \mathrm{MHz}, \Delta v 1,1 \mathrm{H} 55 \mathrm{Mn}=103.8 \mathrm{MHz}$ and $\tilde{v}$ 2, $1 \mathrm{H} 55 \mathrm{Mn}=-148.3 \mathrm{MHz}, \Delta v 2,1 \mathrm{H} 55 \mathrm{Mn}=102.0 \mathrm{MHz}$.

It should be noted, that ${ }^{1} \mathrm{H}-{ }^{55} \mathrm{Mn}$ combination frequencies are also centered around the ${ }^{1} \mathrm{H}$ sq peaks. Equations $1.19-1.22$ show that two combination frequencies which are centered around one sq transition are connected to different allowed EPR transitions ( $\nu_{E P R} \mid 2, \alpha$ or $\left.V_{E P R} \mid 2, \beta\right)$. Therefore, combination frequencies centered around a ${ }^{1} \mathrm{H}$ sq line must be connected to allowed EPR transitions within different nuclear ${ }^{55} \mathrm{Mn}$ spin manifolds. As the ${ }^{55} \mathrm{Mn}$ nucleus is, however, strongly coupled, only one allowed EPR transition within a specific nuclear ${ }^{55} \mathrm{Mn}$ spin manifold can be excited at a time (e.g. $m_{1,1}=\beta$ for the theoretical model presented in this section). As a consequence, only 'one half' of the combination frequencies that are centered around a ${ }^{1} \mathrm{H}$ sq line are excited. The 'second half' will get excited once the allowed EPR transition in the other nuclear spin manifold of the strongly coupled nuclei gets excited (e.g. $m_{\mathrm{l}, 1}=\alpha$. For $\mathrm{Mn}^{2+}$ this corresponds to moving the magnetic field from the third to the fourth hyperfine peak). 
Due to strong level mixing of nuclear spin states, the ${ }^{17} \mathrm{O}$-labeled hexaaqua complex is characterized by very intense ${ }^{17} \mathrm{O}-55 \mathrm{Mn}$ and ${ }^{1} \mathrm{H}-{ }^{17} \mathrm{O}$ combination frequencies. For ${ }^{17} \mathrm{O}-5{ }^{5} \mathrm{Mn}$, only the splitting with the additive hyperfine coupling centered around $-113.3 \mathrm{MHz}$ is clearly resolved $(\Delta v 1,17055 \mathrm{Mn}=21.7 \mathrm{MHz}, \tilde{v} 1,17055 \mathrm{Mn}=-113.4 \mathrm{MHz})$. The splitting with the subtractive hyperfine coupling is not clearly visible, but rather leads to a broadening of the ${ }^{55} \mathrm{Mn}$ sq peak at $-156.4 \mathrm{MHz}$. ${ }^{1} \mathrm{H}-{ }^{17} \mathrm{O}$ combination peaks are centered around the proton signal at $-51.3 \mathrm{MHz}\left(\Delta v_{1 \mathrm{H} 170}=20.6 \mathrm{MHz}, \quad \tilde{v}\right.$ $\left.{ }_{1 \mathrm{H} 170}=-50.9 \mathrm{MHz}\right)$. As one of the ${ }^{1} \mathrm{H}-{ }^{17} \mathrm{O}$ combination signals overlaps with a ${ }^{1} \mathrm{H}-{ }^{55} \mathrm{Mn}$ signal, this may lead to some uncertainties in the exact frequency determination. ${ }^{17} \mathrm{O}$ $\Delta v$ values are, however, in very good agreement with the theoretical value of $2 v_{170}+A_{170}=21.3 \mathrm{MHz}$.

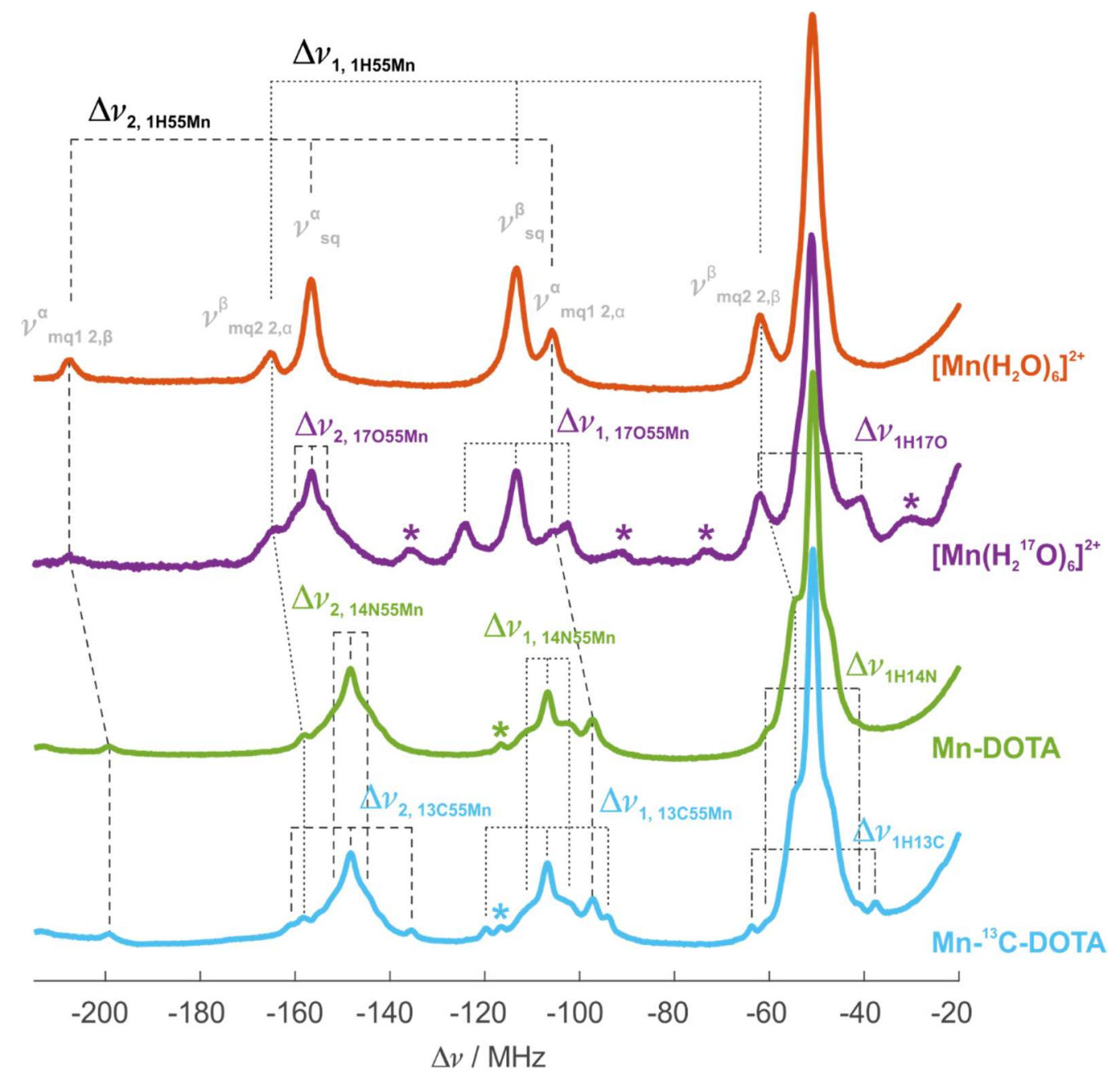

Figure 4: Q-band left-hand side EDNMR spectra of $\left[\mathrm{Mn}\left(\mathrm{H}_{2} \mathrm{O}\right)_{6}\right]^{2+}$ (orange), $\left[\mathrm{Mn}\left(\mathrm{H}_{2}{ }^{17} \mathrm{O}\right)_{6}\right]^{2+}$ (purple), Mn-DOTA (green) and $\mathrm{Mn}-{ }^{13} \mathrm{C}$-DOTA (blue) with higher-powered ELDOR pulses $\left(\omega E L D=8.2 \times 10^{7} \mathrm{rad} \mathrm{s}^{-1}\right)$ to resolve various combination frequencies. ${ }^{55} \mathrm{Mn}$ signals appear around -150 and $-110 \mathrm{MHz}$, whereas weakly coupled protons are centered around $-v_{1 H}=-51 \mathrm{MHz}$. Various combination frequencies of ${ }^{1} \mathrm{H},{ }^{13} \mathrm{C},{ }^{14} \mathrm{~N}$ and ${ }^{17} \mathrm{O}$ with ${ }^{55} \mathrm{Mn}$ are visible, which are centered around one of the ${ }^{55} \mathrm{Mn}$ sq signals. Dotted lines: Combination frequencies centered around the ${ }^{55} \mathrm{Mn}$ signal at $-110 \mathrm{MHz}\left({ }^{\beta} \mathrm{sq}\right)$. Dashed lines: Combination frequencies centered around the ${ }^{55} \mathrm{Mn}$ signal at $-150 \mathrm{MHz}\left(\nu_{s q}^{\alpha}\right)$. Dash-dotted line: Combination frequencies of ${ }^{13} \mathrm{C},{ }^{14} \mathrm{~N}$ and ${ }^{17} \mathrm{O}$ with ${ }^{1} \mathrm{H}$, centered around 
$-v_{1 H}=-51 \mathrm{MHz}$. The expressions above the EDNMR spectrum of $\left[\mathrm{Mn}\left(\mathrm{H}_{2} \mathrm{O}\right)_{6}\right]^{2+}$ correspond to transitions shown in Figure 3 and the analytical expressions given in Equation $1.19-1.24$, although the analytical expressions do not take into account high-spin effects and higher order effects that are necessary to account for the large ${ }^{55} \mathrm{Mn}$ hyperfine coupling [40]. Asteriks denote combination frequencies of the type $\Delta m_{S}= \pm 1, \Delta m_{l, 1}= \pm 1, \Delta m_{l, 2}= \pm 2$. Larmor frequencies of other relevant nuclei: $v_{170}=6.9 \mathrm{MHz}, I=5 / 2 ; v_{14 \mathrm{~N}}=3.7 \mathrm{MHz}, I=1 ; v_{13 \mathrm{C}}=12.8 \mathrm{MHz}, I=1 / 2$.

The EDNMR spectrum of Mn-DOTA exhibits combination signals from ${ }^{1} \mathrm{H}-{ }^{55} \mathrm{Mn}$, ${ }^{14} \mathrm{~N}-{ }^{55} \mathrm{Mn}$ and ${ }^{1} \mathrm{H}-{ }^{14} \mathrm{~N}$. Due to the small ${ }^{14} \mathrm{~N}$ Larmor frequency $(\nu 14 \mathrm{~N}=3.7 \mathrm{MHz}, I=1)$, the ${ }^{14} \mathrm{~N}-{ }^{55} \mathrm{Mn}$ and ${ }^{1} \mathrm{H}-{ }^{14} \mathrm{~N}$ combination signals are not clearly resolved and lead to an apparent line broadening of the ${ }^{55} \mathrm{Mn}$ sq signals (and to some extent to broadening of the ${ }^{1} \mathrm{H}$ signals). The signals of the splitting with the additive hyperfine coupling can be roughly estimated to $\Delta v 1,14 \mathrm{~N} 55 \mathrm{Mn}=8.6 \mathrm{MHz}, \quad \tilde{v} 1,14 \mathrm{~N} 55 \mathrm{Mn}=-106.7 \mathrm{MHz}$. The experimental splitting is in good agreement with the expected value of $2 v_{14 \mathrm{~N}}+A_{14 \mathrm{~N}}=9.1 \mathrm{MHz}$. Upon closer investigation, two satellites signals, centered around the proton signal at $-50.8 \mathrm{MHz}$ become visible. We attribute these satellites signals to combinations frequencies of the type $\Delta m_{\mathrm{s}}= \pm 1, \Delta m_{\mathrm{l}, 1}= \pm 1, \Delta m_{1,2}= \pm 2$ as they agree well with the expected value of $4 v_{14 N}+2 A_{14 N}=18.2 \mathrm{MHz}$ $\left(\Delta v_{1 H} 14 \mathrm{~N}=19.0 \mathrm{MHz}, \tilde{v} 1 \mathrm{H} 14 \mathrm{~N}=-50.9 \mathrm{MHz}\right)$.

In the EDNMR spectrum of $\mathrm{Mn}-{ }^{13} \mathrm{C}-D O T A,{ }^{13} \mathrm{C}-{ }^{55} \mathrm{Mn}$ and ${ }^{1} \mathrm{H}-{ }^{13} \mathrm{C}$ combination frequencies are clearly visible. As the Larmor frequency of ${ }^{13} \mathrm{C}$ is relatively large, the additive $(\Delta v 1,13 \mathrm{C} 55 \mathrm{Mn})$ and subtractive splitting $(\Delta v 2,13 \mathrm{C} 55 \mathrm{Mn})$ are both clearly resolved. The additive splitting is centered around the ${ }^{55} \mathrm{Mn}$ sq transition at $-106.9 \mathrm{MHz}$ $(\Delta v 1,13 \mathrm{C} 55 \mathrm{Mn}=25.8 \mathrm{MHz}, \tilde{v} 1,17055 \mathrm{Mn}=-106.9 \mathrm{MHz})$, whereas the subtractive splitting is centered around the ${ }^{55} \mathrm{Mn}$ sq transition at $-148.3 \mathrm{MHz}(\Delta v 2,13 \mathrm{c} 55 \mathrm{Mn}=25.2 \mathrm{MHz}, \tilde{v}$ $2,17055 \mathrm{Mn}=-148.2 \mathrm{MHz}) \cdot{ }^{1} \mathrm{H}-{ }^{13} \mathrm{C}$ combination frequencies are centered around the proton signals at $-50.8 \mathrm{MHz}\left(\Delta v_{1 \mathrm{H} 13 \mathrm{C}}=26.0 \mathrm{MHz}, \tilde{v_{1}} \mathrm{H}_{13 \mathrm{C}}=-50.7 \mathrm{MHz}\right)$.

Signals marked with an asterisk were assigned to transitions of the type $\Delta m_{\mathrm{S}}= \pm 1$, $\Delta m_{\mathrm{l}, 1}= \pm 1$ and $\Delta m_{\mathrm{l}, 2}= \pm 2$. All these signals match the expected value of $4 u+2 A_{\iota}$ rather well.

\subsection{EDNMR versus Mims ENDOR at Q-band}

${ }^{1} \mathrm{H}$ and ${ }^{14} \mathrm{~N}$ hyperfine interactions of Gd-DOTA and Mn-DOTA were recently investigated with pulsed ENDOR studies at W-band frequencies to draw conclusions about the electron spin delocalization over ligand nuclei $[28,46]$. In the following section, we evaluate the ${ }^{13} \mathrm{C}$-hyperfine interaction of $\mathrm{Mn}-{ }^{13} \mathrm{C}$-DOTA using Mims ENDOR with variable tau $(\tau)$ values and an EDNMR experiment. 

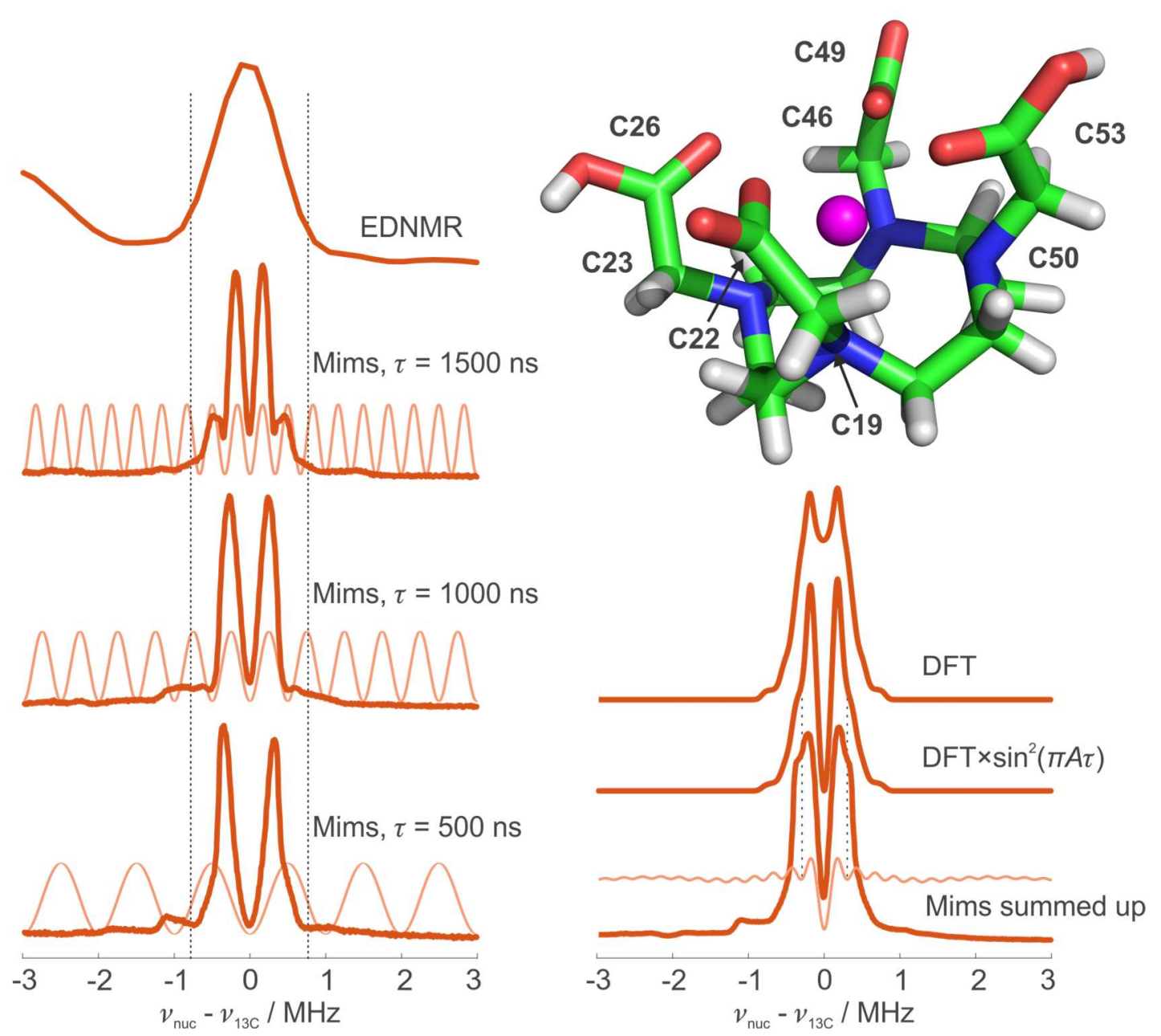

Figure 5: Left: Comparison of the ${ }^{13} \mathrm{C}$ region of a Q-band EDNMR spectrum of Mn- ${ }^{13} \mathrm{C}-D O T A$ with ${ }^{13} \mathrm{C}-\mathrm{Mims}$ ENDOR recorded on the same compound using different tau values. Mims ENDOR blind spot functions of the type $\sin ^{2}(\pi A \tau)=$ $\sin ^{2}\left(\pi\left(2\left(v_{n u c}-v_{13}\right)\right) \tau\right)$ are represented as pale lines. Top right: Crystal structure of Mn-DOTA [36]. The ${ }^{13} \mathrm{C}$-labeled carbon atoms as used in this study are marked. Bottom right: Simulated ENDOR spectrum $(S=1 / 2)$ using the EasySpin toolbox [47] with parameters obtained from DFT calculations on the crystal structure (top trace) compared with a summed Mims ENDOR versus tau $(\tau)$ experiment (bottom trace). The spectrum in the middle represents the simulated spectrum multiplied with the sum of the Mims blind spot functions used in the Mims ENDOR vs tau experiment. The 'dent' in the experimentally recorded Mims ENDOR spectrum at $\pm 0.25 \mathrm{MHz}$ comes from incomplete averaging of the blind spots. The sum of the Mims ENDOR blind spot functions are plotted as a pale line. Hyperfine values obtained from DFT calculations ([aiso, T]) for the labeled ${ }^{13} \mathrm{C}$ positions are: C19, C46 [-0.13, 0.65], C22, C49 $[0.24,0.79], C 23, C 50[-0.05,0.58], C 26, C 53[0.09,0.50]$.

Figure 5 shows a series of Mims ENDOR spectra with different tau values compared to a Q-band ${ }^{13} \mathrm{C}$-EDNMR spectrum. Although EDNMR is more sensitive than ENDOR, information about the spectral line shape is lost in EDNMR, as the canonical orientations (the turning points of the Pake pattern) exhibit a zero transition probability for the forbidden transitions. The disadvantage of Mims ENDOR are blind spots that are defined by an oscillatory function of the type $\sin ^{2}(\pi A \tau)$, where $A$ is the hyperfine coupling and $\tau$ is the first delay time used in the Mims ENDOR pulse sequence. The Mims ENDOR spectrum collected with $\tau=500 \mathrm{~ns}$ is characterized by a rather broad central blind spot. Other blind spots do not distort the intense signals of the nuclear 
frequencies in the two central electron spin manifolds. As a consequence, the flanks of the signals at $\pm 0.3 \mathrm{MHz}$ are reminiscent as edges of a smeared out ENDOR Pake pattern. The signal at $-1 \mathrm{MHz}$ is attributed to nuclear transitions associated with the outer $m s=-3 / 2$ electron spin manifold. Upon increasing $\tau$, more intensity starts to build up around $v_{\text {nuc }}-v_{13 c}=0 \mathrm{MHz}$. Information about the spectral line shape is, however, lost, as now the periodic blind spots are closer together with increasing $\tau$.

In order to support our experimental results, we performed DFT calculations on a Mn-DOTA complex (see Section 3.3 for more details). The calculations yielded small hyperfine couplings, dominated by a dipolar hyperfine coupling of $T=0.50-0.65 \mathrm{MHz}$ and an isotropic contribution close to zero (see Figure 5 right and legend for more information). The deviations from an axial hyperfine tensor were rather small ( $\rho \approx 0.05$, $\rho$ denotes the rhombic component of the hyperfine tensor). Based on the hyperfine values obtained from DFT calculations an ENDOR spectrum was simulated using the EasySpin toolbox [47] (top spectra on the right side of Figure 5). The simulated ENDOR spectrum was compared to the sum of the experimentally recorded ENDOR spectra (bottom trace of Figure 5). The sum of the experimental ENDOR spectra contains artefacts from an incomplete blind spot averaging, as can be seen when examining the sum of the Mims ENDOR blind spot functions (pale lines in Figure 5). In addition, the sum of the experimental ENDOR spectra also contains a central blind spot. In order to compare the experimental data to the simulated data the calculated DFT spectrum was multiplied with the sum of the Mims ENDOR blind spot functions corresponding to the experimental data sets measured. The resulting spectrum is shown as the middle spectrum in Figure 5 on the right side. The line shape is now much more akin to the sum of the experimentally recorded spectra shown at the bottom right of Figure 5.

It should be noted that multiplication of the sum of the Mims blind spot functions (suppression function) with the calculated DFT spectrum is a simplified assumption: The suppression function is always zero at center frequency and so will be the new function after multiplication. This is, however, clearly not correct when looking at the sum of the experimentally recorded Mims ENDOR with different $\tau$ values, shown as the bottom right trace in Figure 5. 


\section{Discussion}

Q-band EDNMR has proven to be a useful technique for detecting nuclear frequencies within Manganese(II) systems and will also be applicable to many other spin active systems. Some points are, however, important to know when recording Q-band EDNMR spectra for low- $\gamma$ nuclei. Firstly; the use of Gaussian ELDOR pulses is very beneficial, as it leads to a narrowing of the central blind spot while maintaining a similar intensity of the forbidden transitions $\left(6^{\text {th }}\right.$ and $7^{\text {th }}$ spectra in Figure 1$)$. Secondly, soft detection pulses should be used to further minimize the width of the central blind spot. In this way, the problem of low- $\gamma$ nuclei being obscured by the central blind spot can be alleviated to some extent.

A problem that is, however, harder to mitigate, is the decreased resolution between nuclear Larmor frequencies of different nuclei at Q-band fields and frequencies. Here, the only solution is a careful planning of sample preparation to remove any nuclei that might overlap with the desired region of study (e.g. substitution of ${ }^{23} \mathrm{Na}$ buffer components with ${ }^{40} \mathrm{~K}$ when investigating ${ }^{13} \mathrm{C}$ resonances with Q-band EDNMR).

In addition a very complicated spectrum is expected when ${ }^{14} \mathrm{~N}$ and ${ }^{17} \mathrm{O}$ are present at the same time. Both nuclei have a small Larmor frequency ( $v_{14 \mathrm{~N}}=3.7 \mathrm{MHz}$ and $v_{170}=6.9 \mathrm{MHz}$ ) but relatively large quadrupole interactions, which leads to intense dq, tq and mq transitions, that are very difficult to disentangle and interpret accurately due to severe overlap of different signals. For ${ }^{14} \mathrm{~N}$ and ${ }^{17} \mathrm{O}$, combination frequencies are visible, even when using relatively low powered ELDOR pulses. In addition, care has to be taken when analyzing the line width of sq transitions, as the sq peaks may be broadened by combination frequencies (e.g. ${ }^{55} \mathrm{Mn}$ ).

Certain aspects, such as a low concentration or small binding affinity may mandate the use of higher powered ELDOR pulses in order to increase the EDNMR signal observed. At Q-band frequencies, this can lead to the appearance of relatively intense combination frequencies, which can easily be misinterpreted as sq signals of other nuclei. Using the first-order expressions given in Equations $1.19-1.24$, we were able to assign all additional signals when using higher powered ELDOR pulses for the four systems studied in this work. In addition, combination frequencies can give an estimation of the magnitude of the hyperfine coupling if analyzed correctly. It is noted, that the first-order equations in Section 4.3 do not reflect a correct physical description as they lack the pseudosecular part of the hyperfine coupling and high-spin interactions 
from the $\mathrm{Mn}^{2+}$ electron and ${ }^{55} \mathrm{Mn}$ nuclear spin. However, they still proved useful in identifying the different combination frequencies present. The underlying physical mechanism for the appearance of the fairly intense combination frequencies remains unknown. It is noted, that for most systems with g-values in the region of $g=2$, Q-band EDNMR is most likely the useful lower limit in terms of microwave frequencies when investigating low- $\gamma$ nuclei $\left(\nu_{14 \mathrm{~N}}=1.1 \mathrm{MHz}\right.$ at $\mathrm{X}$-band). For these systems at $\mathrm{X}$-band, the blind spot separation and the resolution of nuclear Larmor frequencies will get significantly worse. Furthermore, even more intense dq, tq and even $\mathrm{mq}$ are expected for some nuclei; for example for ${ }^{14} \mathrm{~N}$ and ${ }^{17} \mathrm{O}$ the cancellation condition is perfectly fulfilled for certain orientations.

The acquisition time for each EDNMR spectra shown in this work was around 20 minutes. In order to achieve a similar signal-to-noise for sub-mM samples (e.g. $0.2 \mathrm{mM}$ ), the acquisition time would be around 2000 minutes ( $\approx 30$ hours). The acquisition time can be reduced by using less selective detection pulses and by constraining the frequency range to one side of the EDNMR spectrum of the nuclei of interest. Therefore, EDNMR spectra for a $0.2 \mathrm{mM}$ sample with a good signal-to-noise ratio and a sufficient resolution are obtainable in $\approx 3$ hours.

Complete characterization of the ${ }^{13} \mathrm{C}$ hyperfine coupling of $\mathrm{Mn}-{ }^{13} \mathrm{C}$-DOTA with Mims ENDOR was difficult, as the hyperfine couplings are rather small and most of the intensity occurs around $v$. In addition, Mims ENDOR spectra could not be freed completely of the central blind spot, even with the collection and summation of data using different tau values. The experimentally recorded summed Mims versus tau spectrum is, however, in good agreement with the blind spot corrected EasySpin simulation using EPR parameters obtained from DFT calculations on the crystal structure of the complex: The maxima at $\pm 0.2 \mathrm{MHz}$ coincide. So does the width of simulated and experimental spectrum and the shape at the flanks of the two intense signals at $\pm 0.5 \mathrm{MHz}$. As expected, the electron spin delocalization over ${ }^{13} \mathrm{C}$ ligand nuclei is therefore negligibly small. The two small features in the simulated spectra at $\pm 0.75 \mathrm{MHz}$ could not be identified in the experimental spectrum, but may not be observed due to incomplete cancellation of the experimental blind-spots. The width of the EDNMR spectrum and the Mims ENDOR spectrum with tau $=500 \mathrm{~ns}$ is also very similar, indicating that i) EDNMR can be used to constrain the magnitude of the hyperfine coupling constant if ii) the EDNMR linewidth is not broadened by to short 
detection pulses, too highly powered ELDOR pulses, or magnetization transfer artefacts due to too long ELDOR pulses (see Figure 1).

\section{Conclusion}

Q-band EDNMR has been shown to be a valuable method to detect nuclear frequencies of low- $\gamma$ with nuclear frequencies of $2.6 \mathrm{MHz}$ upwards. Although the principal values of the hyperfine tensor could not be determined due to vanishingly small transition probabilities at the canonical orientations, estimations of the magnitude of the hyperfine coupling could be determined from dq $\left({ }^{14} \mathrm{~N}\right)$ or resolved sq $\left({ }^{17} \mathrm{O}\right)$ splittings. For our $\mathrm{Mn}^{2+}$ systems, highly selective detection pulses (400-800 ns or 200-400 ns) and an ELDOR pulse with an amplitude of $\omega E L D \approx 1.0 \times 10^{7} \mathrm{rad} \mathrm{s}^{-1}$ gave the best results in terms of balancing signal intensity with a narrow central blind spot. An advantage of Q-band EDNMR over higher frequencies is its high sensitivity, as the transition probability of the forbidden transitions upon which the technique relies is intrinsically higher than at higher microwave frequencies. This can be a particular advantage when performing 2D-experiments such as THYCOS [12] or 2D-EDNMR [14], e.g. between ${ }^{13} \mathrm{C}$ and ${ }^{31} \mathrm{P}$.

\section{Acknowlegements}

This work was supported by the Deutsche Forschungsgemeinschaft (CRC 902 Molecular Principles of RNA-based Regulation). AMB acknowledges the Goethe International (GOIN) postdoctoral fellowship program and the Royal Society - EPSRC Dorothy Hodgkin fellowship program for generous support. We are grateful to Prof. Christiane Timmel and Dr William Myers at the Centre of Advanced Electron Spin Resonance (CAESR), Oxford University for access to their facilities for some of the aforementioned experiments.

\section{References}

1. P. Schosseler, T. Wacker, and A. Schweiger, Chem. Phys. Lett. 224, 319 (1994).

2. G. Jeschke and H. W. Spiess, Chem. Phys. Lett. 293, 9 (1998).

3. H. Mino and T. Ono, Appl. Magn. Reson 23, 571 (2003).

4. L. Kulik, B. Epel, J. Messinger, and W. Lubitz, Photosynth. Res. 84, 347 (2005).

5. N. Cox, W. Lubitz, and A. Savitsky, Mol. Phys. 111, 2788 (2013). 
6. N. Cox, A. Nalepa, M. E. Pandelia, W. Lubitz, and A. Savitsky, in Methods Enzymol., 1st ed. (Elsevier Inc., 2015), pp. 211-249.

7. D. Goldfarb, eMagRes 6, 101 (2017).

8. N. Cox, A. Nalepa, W. Lubitz, and A. Savitsky, J. Magn. Reson. 280, 63 (2017).

9. S. Un, Inorg. Chem. 52, 3803 (2013).

10. E. M. Bruch, M. T. Warner, S. Thomine, L. C. Tabares, and S. Un, J. Phys. Chem. B 119, 13515 (2015).

11. S. Un and E. M. Bruch, Inorg. Chem. 54, 10422 (2015).

12. A. Potapov, B. Epel, and D. Goldfarb, J. Chem. Phys. 128, (2008).

13. M. Florent, I. Kaminker, V. Nagarajan, and D. Goldfarb, J. Magn. Reson. 210, 192 (2011).

14. I. Kaminker, T. D. Wilson, M. G. Savelieff, Y. Hovav, H. Zimmermann, Y. Lu, and D. Goldfarb, J. Magn. Reson. 240, 77 (2014).

15. M. Ramirez Cohen, N. Mendelman, M. Radoul, T. D. Wilson, M. G. Savelieff, H. Zimmermann, I. Kaminker, A. Feintuch, Y. Lu, and D. Goldfarb, Inorg. Chem. 56, 6163 (2017).

16. L. Rapatskiy, N. Cox, A. Savitsky, W. M. Ames, J. Sander, M. M. Nowaczyk, M. Rögner, A. Boussac, F. Neese, J. Messinger, and W. Lubitz, J. Am. Chem. Soc. 134, 16619 (2012).

17. A. Nalepa, K. Möbius, W. Lubitz, and A. Savitsky, J. Magn. Reson. 242, 203 (2014).

18. M. Fittipaldi, I. García-Rubio, F. Trandafir, I. Gromov, A. Schweiger, A. Bouwen, and S. Van Doorslaer, J. Phys. Chem. B 112, 3859 (2008).

19. S. Zamani, V. Meynen, A.-M. Hanu, M. Mertens, E. Popovici, S. Van Doorslaer, and P. Cool, Phys. Chem. Chem. Phys. 11, 5823 (2009).

20. N. V. Nagy, S. Van Doorslaer, T. Szabó-Plánka, S. Van Rompaey, A. Hamza, F. Fülöp, G. K. Tóth, and A. Rockenbauer, Inorg. Chem. 51, 1386 (2012).

21. M. Flores, A. G. Agrawal, M. Van Gastel, W. Gärtner, and W. Lubitz, J. Am. Chem. Soc. 130, 2402 (2008). 
22. S. Van Doorslaer and E. Vinck, Phys. Chem. Chem. Phys. 9, 4620 (2007).

23. A. Aliabadi, R. Zaripov, K. Salikhov, V. Voronkova, E. Vavilova, M. A. Abdulmalic, T. Rueffer, B. Buechner, and V. Kataev, J. Phys. Chem. B 119, 13762 (2015).

24. A. Schweiger and G. Jeschke, Principles Of Pulse Electron Paramagnetic Resonance (Oxford University Press, Oxford, 2001).

25. B. Bleaney and R. S. Rubins, Proc. Phys. Soc. 77, 103 (1961).

26. E. Meirovitch and R. Poupko, J. Phys. Chem. 82, 1920 (1978).

27. O. Schiemann, R. Carmieli, and D. Goldfarb, Appl. Magn. Reson. 31, 543 (2007).

28. K. Keller, M. Zalibera, M. Qi, V. Koch, J. Wegner, H. Hintz, A. Godt, G. Jeschke, A. Savitsky, and M. Yulikov, Phys. Chem. Chem. Phys. 18, 25120 (2016).

29. A. Collauto, S. Mishra, A. Litvinov, H. S. Mchaourab, and D. Goldfarb, Structure 25, 1264 (2017).

30. J. F. Desreux, Inorg. Chem. 19, 1319 (1980).

31. L. Lumata, M. Merritt, C. Malloy, A. D. Sherry, and Z. Kovács, Appl. Magn. Reson. 43, 69 (2012).

32. B. Epel, D. Arieli, D. Baute, and D. Goldfarb, J. Magn. Reson. 164, 78 (2003).

33. F. Neese, Wiley Interdiscip. Rev. Comput. Mol. Sci. 2, 73 (2012).

34. C. Adamo and V. Barone, J. Chem. Phys. 110, 6158 (1999).

35. V. Barone and M. Cossi, J. Phys. Chem. A 102, 1995 (1998).

36. S. Wang and T. D. Westmoreland, Inorg. Chem. 48, 719 (2009).

37. T. Wacker, G. A. Sierra, and A. Schweiger, Isr. J. Chem. 32, 305 (1992).

38. H. Y. Vincent Ching, P. Demay-Drouhard, H. C. Bertrand, C. Policar, L. C. Tabares, and S. Un, Phys. Chem. Chem. Phys. 17, 23368 (2015).

39. S. Sinnecker, F. Neese, L. Noodleman, and W. Lubitz, J. Am. Chem. Soc. 126, 2613 (2004).

40. B. E. Sturgeon, J. A. Ball, D. W. Randall, and R. D. Britt, J. Phys. Chem. 98, 12871 (1994). 
41. P. Manikandan, R. Carmieli, T. Shane, A. J. Kalb, and D. Goldfarb, J. Am. Chem. Soc. $122,3488(2000)$.

42. X. Tan, M. Bernardo, H. Thomann, and C. P. Scholes, J. Chem. Phys. 102, 2675 (1995).

43. D. Baute and D. Goldfarb, J. Phys. Chem. A 109, 7865 (2005).

44. F. E. Mabbs and D. Collison, in Stud. Inorg. Chem. (1992), pp. 189-217.

45. H. L. Flanagan and D. J. Singel, J. Chem. Phys. 87, (1987).

46. A. Collauto, A. Feintuch, M. Qi, A. Godt, T. Meade, and D. Goldfarb, J. Magn. Reson. 263, 156 (2016).

47. S. Stoll and A. Schweiger, J. Magn. Reson. 178, 42 (2006). 